\title{
Adjoint-Based Optimization of PDE Systems with Alternative Gradients
}

\author{
Bartosz Protas $^{\mathrm{a}}$ \\ ${ }^{a}$ Department of Mathematics \& Statistics, McMaster University, Hamilton, Ontario, \\ Canada
}

\begin{abstract}
In this work we investigate a technique for accelerating convergence of adjoint-based optimization of PDE systems based on a nonlinear change of variables in the control space. This change of variables is accomplished in the differentiate-then-discretize approach by constructing the descent directions in a control space not equipped with the Hilbert structure. We show how such descent directions can be computed in general Lebesgue and Besov spaces, and argue that in the Besov space case determination of descent directions can be interpreted as nonlinear wavelet filtering of the adjoint field. The freedom involved in choosing parameters characterizing the spaces in which the steepest descent directions are constructed can be leveraged to accelerate convergence of iterations. Our computational examples involving state estimation problems for the 1D Kuramoto-Sivashinsky and 3D Navier-Stokes equations indeed show significantly improved performance of the proposed method as compared to the standard approaches.
\end{abstract}

Key words: optimal control, adjoint equations, state estimation, preconditioning, computational fluid dynamics

PACS: 47.27.Rc, 47.27.nd, 47.10.A-

\section{Introduction}

Problems of optimal control of distributed systems arise in many areas of science and engineering. Without loss of generality, in this investigation we will focus on problems motivated by applications in fluid mechanics such as:

- shape optimization in aerodynamics (see, e.g., [1,2]),

- flow control for drag reduction, (see, e.g., $[3,4]$ ),

- variational data assimilation in dynamic meteorology known as 4DVAR (see, e.g., [5]),

- mixing enhancement (see, e.g., [6]). 
Denoting the state of the system $\mathbf{v} \in \mathcal{X}$, where $\mathcal{X}$ is an infinite dimensional state space, and the control variable $\phi \in \mathcal{U}$, where $\mathcal{U}$ is a finite-dimensional or infinitedimensional control space, the governing system of partial differential equations (PDEs) can be expressed in a general form as $\mathcal{G}(\mathbf{v}, \phi)=0$. Then the problem of finding an optimal control $\phi_{o p t}$ can be stated as PDE-constrained optimization in the following way

$$
\begin{aligned}
& \min _{\mathbf{v} \in \mathcal{X}, \phi \in \mathcal{U}} j(\mathbf{v}, \phi) \\
& \text { subject to } \mathcal{G}(\mathbf{v}, \phi)=0,
\end{aligned}
$$

where $j: \mathcal{X} \times \mathcal{U} \rightarrow \mathbb{R}$ is the cost functional representing mathematically the performance criterion we want to optimize. In principle, after a suitable discretization, problems of the type (1) can be solved using general methods of Nonlinear Programming (NLP), see, e.g., [7]. However, from the computational point of view, formulation (1) is not quite convenient when dealing with distributed systems, as it requires simultaneous optimization over discretizations of the state and control spaces $\mathcal{X}$ and $\mathcal{U}$, the first of which may have a very large dimension. In practice, in the presence of equality constraints only and subject to certain assumptions on the function $\mathcal{G}(\mathbf{v}, \phi)$, we can write $\mathbf{v}=\mathbf{v}(\phi)$ which allows us to define the reduced cost functional $\mathcal{I}(\phi) \triangleq j(\mathbf{v}(\phi), \phi)$, so that (1) can be transformed to the equivalent unconstrained formulation

$$
\min _{\phi \in \mathcal{U}} \mathcal{I}(\phi) .
$$

The advantage of (2) as compared to (1) is that now optimization must be performed with respect to the control variable $\phi$ only. Hereafter we will focus exclusively on formulation (2) and, unless needed for clarity, we will drop the adjective "reduced" when referring to the cost functional $\mathcal{I}(\phi)$. The mathematical theory of optimal control of PDE systems was developed initially by Lions [8]; in the context of problems arising in fluid mechanics it was further investigated by Abergel and Temam [9]. The state of the art of this area is surveyed in the monograph by Gunzburger [10]. There are two main paradigms relevant to solving problem (2): the "discretize-then-differentiate" approach stipulates that the state variable $\mathbf{v}$ and, if necessary, the control variable $\phi$ are discretized first and then the optimization problem is solved in the finite-dimensional setting; on the other hand, in the "differentiate-then-discretize" approach the optimality conditions are derived on the continuous (PDE) level and only then discretized and solved numerically. While it is recognized that in general these two routes may lead to different results and there is an on-going debate as to the relative merits of the two approaches [10], in the present work we will adopt the "differentiate-then-discretize" methodology, as in this context our approach is more straightforward to introduce.

There are aspects of optimal control problems in fluid mechanics that make their computational solution particularly challenging. First of all, given the usual dimension of spatio-temporal discretizations $\tilde{\mathbf{v}}$ of the state variable $\mathbf{v}$ [tilde ( $\sim$ ) will denote a discretized version of a variable, operator, or space], which often involve as 
many as $O\left(10^{16}\right)$ degrees of freedom, it is impossible to store the linear operators acting on $\tilde{\mathbf{v}}$ as matrices. Therefore most existing NLP software packages may not be used and "matrix-free" alternatives must be developed. Moreover, complete Hessian information is often unavailable and consequently Newton's method can rarely be used. Consequently, one must employ quasi-Newton or gradient approaches and in this investigation we focus on gradient-based methods. The necessary condition characterizing the minimizer $\phi_{\text {opt }}$ of the cost functional $\mathcal{I}(\phi)$ is the vanishing of the Gâteaux differential $\mathcal{I}^{\prime}: \mathcal{U} \times \mathcal{U} \rightarrow \mathbb{R}$, i.e.,

$$
\mathcal{I}^{\prime}\left(\phi_{o p t} ; \phi^{\prime}\right)=0, \forall \phi^{\prime} \in \mathcal{U}
$$

where the Gâteaux differential is defined as $\mathcal{I}^{\prime}\left(\phi ; \phi^{\prime}\right) \triangleq \lim _{\varepsilon \rightarrow 0} \frac{\mathcal{J}\left(\phi+\varepsilon \phi^{\prime}\right)-\mathcal{g}(\phi)}{\varepsilon}$ (the symbol $\triangleq$ means “equal to by definition"). In many applications, including the cases considered in the present work, the cost functional $j$ is quadratic in both $\mathbf{v}$ and $\phi$, however, $\mathbf{v}=\mathbf{v}(\phi)$ is a nonlinear mapping, and therefore optimization problem (2) will often be nonconvex. As a result, problem (2) may admit several local minimizers and (3) will characterize such a local minimizer $\phi_{\text {opt }}$ only. Given a discretization of some initial guess $\tilde{\phi}^{(0)}=\tilde{\phi}_{0}$, an approximation of a minimizer can be found using a gradient-based descent method of the general form

$$
\tilde{\phi}^{(n+1)}=\tilde{\phi}^{(n)}+\mathcal{A} \widetilde{\nabla \mathcal{I}}\left(\tilde{\phi}^{(n)}\right), \quad n=0,1, \ldots,
$$

such that $\lim _{n \rightarrow \infty} \tilde{\phi}^{(n)}=\tilde{\phi}_{\text {opt }}$, where $n$ is the iteration count. At every iteration $n$ the descent direction $\mathscr{A} \widetilde{\nabla \mathcal{I}}$ is determined based on the gradient $\widetilde{\nabla \mathcal{I}}$ of the cost functional evaluated at $\tilde{\phi}^{(n)}$ and iterations are performed until a critical point is reached, i.e., until $\widetilde{\nabla g}\left(\tilde{\phi}^{(n)}\right)=0$ in some approximate sense. As will be shown below, this gradient can be conveniently expressed using solutions of a suitablydefined adjoint system. Adopting different forms of the operator $\mathcal{A}$ we may recover different variants of the gradient method such as the steepest descent method, the conjugate gradient method, etc.

We emphasize that, as shown in [11], PDE-constrained optimization problems with quadratic cost functionals are often of the elliptic type in the sense that the reduced Hessian of the cost functional is an elliptic operator regardless of the specific type of the PDE representing the constraint. It is also well known $[7,12]$ that for convex problems the rate of convergence of gradient iterations (4) to the discrete minimizer $\tilde{\phi}_{\text {opt }}$ depends on the condition number $\varkappa$ of the (reduced) Hessian of $\mathcal{J}(\tilde{\phi})$ in the neighborhood of the minimizer. The condition number characterizes the local ellipticity of the isocontours of $\mathcal{I}(\tilde{\phi})$, i.e., when $\varkappa \gtrsim 1$ these isocontours are close to spheres, whereas when $\varkappa \gg 1$, the isocontours are squashed in some directions resulting in many iterations required in order to converge to the minimizer in (4). Convergence of gradient iterations (4) can thus be accelerated by a suitable rescaling of the independent variables, so that the condition number of the problem is reduced, a procedure known as preconditioning. The effect of preconditioning can 
be represented as multiplication of the descent direction $\mathscr{A} \widetilde{\nabla g}$ in (4) by a preconditioning operator (matrix) $\tilde{\mathcal{T}}$ chosen so that the condition number of the resulting problem is reduced. Algebraic preconditioning strategies [13] determine the preconditioner $\tilde{\mathcal{T}}$ by exploiting the algebraic structure of the descent direction $\mathscr{A} \tilde{\nabla \mathcal{g}}$. On the other hand, operator preconditioning strategies [14] determine a continuous preconditioning operator $\mathcal{T}$ on the PDE level by analyzing properties of the infinite-dimensional problem. An advantage of the latter approach is that the resulting improvements in the bounds on the condition numbers are often discretizationindependent. An example of the operator preconditioning approach is furnished by the method of Sobolev gradients developed by Neuberger [15] which derives the preconditioning operators from the corresponding Sobolev norms. In other words, when using different Hilbert spaces $\mathcal{V}$ equipped with the inner products $(\cdot, \cdot)_{\mathcal{V}}$ as the control space $\mathcal{U}$, Riesz' theorem [16] guarantees existence of the corresponding gradients $\nabla^{\mathcal{V}} \mathfrak{g} \in \mathcal{V}$ which are defined through the Riesz identity

$$
\mathcal{I}^{\prime}\left(\phi ; \phi^{\prime}\right)=\left(\nabla^{\mathcal{V}} \mathcal{J}, \phi^{\prime}\right)_{\mathcal{V}}, \quad \forall_{\phi^{\prime} \in \mathcal{V}}
$$

As will be shown below, different Hilbert (Sobolev) gradients can be computed by applying a linear transformation to the adjoint field. An approach employing Sobolev spaces $H^{p}$ as the Hilbert spaces for gradient extraction was used with success for solution of problems involving minimization of potentials [17] and was also employed in the context of PDE-constrained optimization in [18]. A simple, yet illuminating, example illustrating an application of operator preconditioning to solution of a linear boundary value problem for an elliptic PDE is presented in Appendix A. The utility of different Hilbert space gradients in an area of mathematical modelling was recently reviewed in [19]. Preconditioners, both of the algebraic and operator type, are also useful for solution of nonlinear problems, however, in such situations the preconditioner is often allowed to vary from one iteration to another, resulting in the so-called variable preconditioning $[13,14]$. This approach is related to the variable metric method used for solution of systems of nonlinear algebraic equations [12]. As regards PDE-constrained optimization, a heuristically motivated variable preconditioning approach was developed in [18], where it was proposed to precondition the gradients by "focusing" them on a specific range of length-scales which was modified during the iterations, thereby resulting in an effectively multiscale strategy.

The goal of the present paper is to propose and investigate a more general preconditioning approach for accelerating convergence of adjoint-based optimization of PDE systems. This method extends the concept of the Sobolev gradients by making it possible to extract their counterparts in general Banach spaces not equipped with the Hilbert structure. Since Riesz representation (5) does not apply in nonHilbert spaces, we employ a more general procedure to extract the steepest descent directions in Banach spaces which follows the proposal first made by Neuberger in [15]. This procedure will involve a nonlinear transformation of the adjoint field equivalent to a nonlinear change of variables in iterative procedure (4). Further- 
more, by extracting the steepest descent directions in a continuous family of nested spaces we will allow this change of the metric to vary in the course of iterations (4). Our computational examples illustrating application of this strategy to solve optimal control problems for two different PDE systems show advantages of the proposed approach with respect to traditional techniques. As argued in [18] and as is also well-known in the image processing literature (see, e.g., [20]), extraction of gradients in different functional spaces is in fact equivalent to applying different filters to the adjoint field. Gradients extracted in Hilbert spaces can be regarded as obtained via an application of a linear filter to the adjoint state and, for example, the Sobolev gradients can be viewed as obtained via application of suitable low-pass filters (defined in wavenumber space) to the adjoint field [18]. In the same spirit, extraction of the steepest descent directions in general Banach spaces not endowed with a Hilbert structure can be regarded as application of a nonlinear filter to the adjoint field. In particular, as will be shown below, identification of directions of the steepest descent in Besov spaces can be regarded as nonlinear wavelet filtering of the adjoint field emphasizing its coherent part [21], in contrast to the low-pass filtering which emphasizes specific wavenumber components only.

The structure of the paper is as follows: in the next Section we introduce our two model PDE-constrained optimization problems, one formulated using the KuramotoSivashinsky equation in a one-dimensional (1D) periodic domain and the other formulated using the Navier-Stokes system in a three-dimensional (3D) channel periodic in the streamwise and spanwise directions; in that Section we also show how the Sobolev gradients of the relevant cost functionals can be identified from solutions of the appropriate adjoint problems; in the following Section we describe how directions of the steepest descent in various Banach spaces (Lebesgue, Besov) can be identified; in Section 4 we present computational results illustrating how these descent directions can be used to accelerate convergence of the iterative solution procedure; summary and conclusions are deferred to Section 5.

\section{Model Optimal Control Problems}

In this Section we set up two model PDE-constrained optimization problems whose computational solution will be used to illustrate our new approach. In both cases we are interested in solving a variational data assimilation problem (4DVAR) [5], wherein, given some incomplete and possibly noisy measurements of the system evolution over the time window $[0, T]$, one seeks to determine the initial condition (the control variable) $\phi$ in such a way that the ensuing system evolution matches the available measurements as well as possible. We formulate this problem for the Kuramoto-Sivashinsky equation in a $1 \mathrm{D}$ periodic domain and then the NavierStokes system in a 3D channel. These problems were introduced as benchmarks for adjoint-based optimization in [18] and [22], respectively. An advantage of using such "synthetic" state estimation problems is that in the absence of noise we 
know the exact minimizer (which is the state used to generate the measurements) and thus we can know whether the minimum actually found is local or global. Without the risk of confusion, in certain cases we will use the same symbols to denote analogous quantities in the statement of the optimization problems for the Kuramoto-Sivashinsky and Navier-Stokes equations in Sections 2.1 and 2.2, respectively.

\subsection{State Reconstruction for the Kuramoto-Sivashinsky Equation}

The Kuramoto-Sivashinsky equation is chosen here, since its solutions are endowed with chaotic and multiscale behavior which makes it an attractive model for the Navier-Stokes system. For simplicity, we will consider this equation on a periodic spatial domain $\Omega=(0,2 \pi)$ and a time interval $[0, T]$

$$
\begin{cases}\partial_{t} u+4 \partial_{x}^{4} u+\kappa\left(\partial_{x}^{2} u+u \partial_{x} u\right)=0, & x \in \Omega, t \in[0, T] \\ \partial_{x}^{i} u(0, t)=\partial_{x}^{i} u(2 \pi, t), & t \in[0, T], i=0, \ldots, 3 \\ u(x, 0)=\phi, & x \in \Omega .\end{cases}
$$

Out of many different ways in which the coefficients of the Kuramoto-Sivashinsky equation may be scaled, we follow here the approach proposed in [23]. In our computations we will set $\kappa=4000$ which will result in 23-24 coherent structures ("bumps") present in the domain. A solution of system (6) exhibiting a characteristic spatio-temporal pattern is shown in Fig. 1 (the numerical method used to obtain this solution is described further below). We refer the reader to [18] for further details related to the Kuramoto-Sivashinsky as a model problem for adjoint-based optimization.

Given incomplete (and possibly noisy) measurements $y=\mathcal{H} u_{a c t} \in \mathcal{Y}$, where $u_{\text {act }}(\cdot, t) \in$ $X$ are states at the actual system trajectory and $\mathcal{H}: X \rightarrow \mathcal{Y}$ is an observation operator with $\mathcal{Y}$ the space of measurements, our optimization problem consists in finding an initial condition $\phi_{o p t}$ in (6) which minimizes the following cost functional

$$
\mathcal{J}(\phi)=\frac{1}{2} \int_{0}^{T}[\mathcal{H} u(\phi)-y]^{2} d \tau
$$

Consistently with the properties of system (6) [24], we will assume that $\phi \in \mathcal{U}=$ $L_{2}(\Omega)$. Since $\mathcal{I}$ depends on the control variable $\phi$ implicitly through state equation (6), expression (7) represents in fact a reduced cost functional. We will assume that the observation operator $\mathcal{H}$ has the form of projection of the state $u$ on a set of cosine modes with the wavenumbers in some set $\Lambda_{r}$, i.e.,

$$
\mathcal{H} \triangleq \sum_{r \in \Lambda_{r}} \mathcal{P}_{r}, \quad \text { where } \quad \mathcal{P}_{r} z \triangleq\left[\frac{1}{\pi} \int_{0}^{2 \pi} \cos \left(r x^{\prime}\right) z\left(x^{\prime}\right) d x^{\prime}\right] \cos (r x)
$$




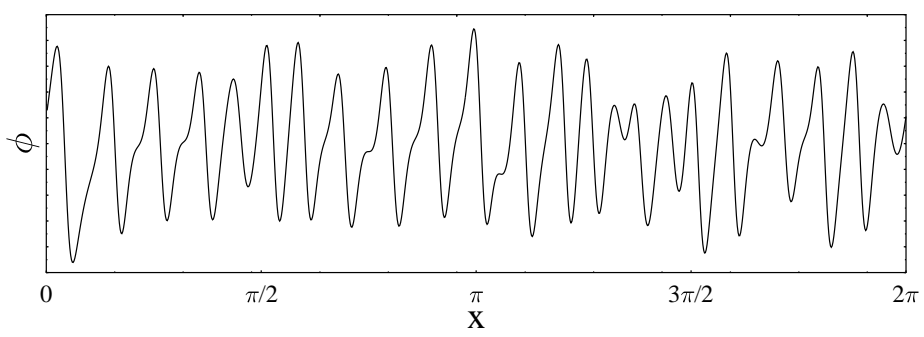

(a)

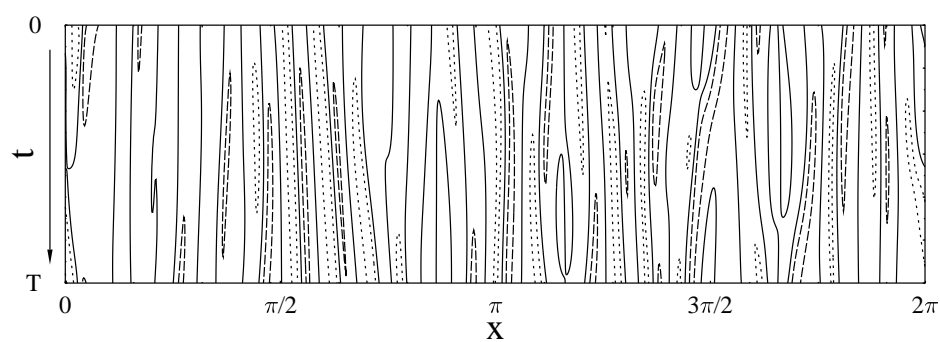

(b)

Fig. 1. Dynamics of the Kuramoto-Sivashinsky system for $\kappa=4 \cdot 10^{3}$ : (a) initial condition $\phi$ (chosen on the chaotic attractor of the system), and (b) spatio-temporal evolution of the system [visualized are the zero (solid), several positive (dotted) and negative (dashed) isocontours of $u$ in the space-time plane].

The Gâteaux differential of (7) is given by

$$
g^{\prime}\left(\phi ; \phi^{\prime}\right)=\int_{0}^{T} \int_{0}^{2 \pi}(\mathcal{H} u-y) \mathcal{H} u^{\prime} d x d t
$$

where the perturbation $u^{\prime}\left(\phi ; \phi^{\prime}\right)$ satisfies the Kuramoto-Sivashinsky equation linearized around the state $u(\phi)$, i.e.,

$$
\begin{cases}\mathcal{L} u^{\prime} \triangleq \partial_{t} u^{\prime}+4 \partial_{x}^{4} u^{\prime}+\kappa\left[\partial_{x}^{2} u^{\prime}+u \partial_{x} u^{\prime}+\left(\partial_{x} u\right) u^{\prime}\right]=0, & x \in \Omega, t \in[0, T], \\ \partial_{x}^{i} u^{\prime}(0, t)=\partial_{x}^{i} u^{\prime}(2 \pi, t), & t \in[0, T], \quad i=0, \ldots, 3, \\ u^{\prime}(x, 0)=\phi^{\prime}, & x \in \Omega,\end{cases}
$$

with the operator $\mathcal{L}: X \rightarrow X^{*}$ understood in the weak sense $\left(X^{*}\right.$ is the space dual to $\mathcal{X}$ ). Relation (9) can now be transformed to a form consistent with (5) by introducing an adjoint operator $\mathcal{L}^{*}: X \rightarrow \mathcal{X}^{*}$ and the corresponding adjoint state $u^{*} \in \mathcal{X}$ via the following identity

$$
\left\langle u^{*}, \mathcal{L} u^{\prime}\right\rangle_{X \times X^{*}}=\left\langle\mathcal{L}^{*} u^{*}, u^{\prime}\right\rangle_{X \times X^{*}}+b
$$

where $\langle\cdot, \cdot\rangle_{X \times X} X^{*}$ represents the duality pairing between the spaces $X$ and its dual $X^{*}$, i.e., given $z_{1} \in X$ and $z_{2} \in \mathcal{X}^{*},\left\langle z_{1}, z_{2}\right\rangle \chi \times X^{*} \triangleq \int_{0}^{T} \int_{0}^{2 \pi} z_{1} z_{2} d t d x$. Using integra- 
tion by parts and the definition of $\mathcal{L}$ in (10), we obtain

$$
\begin{aligned}
& \mathcal{L}^{*} u^{*} \triangleq-\partial_{t} u^{*}+4 \partial_{x}^{4} u^{*}+\kappa\left(\partial_{x}^{2} u^{*}-u \partial_{x} u^{*}\right), \quad \text { and } \\
& b=\left[\int_{0}^{2 \pi} u^{*} u^{\prime} d x\right]_{t=0}^{t=T} .
\end{aligned}
$$

We remark that $b$ does not contain any boundary terms (resulting from integration by parts), since all of them vanish due to periodicity. Defining an adjoint system as

$$
\begin{cases}\mathcal{L}^{*} u^{*}=\mathcal{H}^{*}(\mathcal{H} u-y), & x \in \Omega, \quad t \in[0, T], \\ \partial_{x}^{i} u^{*}(0, t)=\partial_{x}^{i} u^{*}(2 \pi, t), & t \in[0, T], \quad i=0, \ldots, 3, \\ u^{*}(x, T)=0, & x \in \Omega,\end{cases}
$$

and using (10), (11) and (12) we can now express Gâteaux differential (9) in the desired form (5), namely

$$
g^{\prime}\left(\phi ; \phi^{\prime}\right)=\left.\int_{0}^{2 \pi} u^{*}\right|_{t=0} \phi^{\prime} d x
$$

Thus, this differential (i.e., the sensitivity of the cost functional $\mathcal{I}$ with respect to perturbations $\phi^{\prime}$ of the initial condition) can be expressed using the solution of adjoint system (13). Physically, the adjoint field evaluated in the part of the domain $\Omega \times[0, T]$ where the control is defined represents the sensitivity of cost functional (7) to the control variable. In the present problem, this control variable is the unknown initial condition which we seek to reconstruct.

Relationship (14) can now be employed to extract the gradient required in descent optimization algorithm (4). Since $\mathcal{U}=L_{2}(0,2 \pi)$ is equipped with the inner product $\left(z_{1}, z_{2}\right)_{L_{2}} \triangleq \int_{0}^{2 \pi} z_{1} z_{2} d x$ for $z_{1}, z_{2} \in L_{2}(0,2 \pi)$, we immediately obtain

$$
\mathcal{g}^{\prime}\left(\phi ; \phi^{\prime}\right)=\left.\int_{0}^{2 \pi} u^{*}\right|_{t=0} \phi^{\prime} d x=\left(\nabla^{L_{2}} \boldsymbol{g}, \phi^{\prime}\right)_{L_{2}} \Longrightarrow \nabla^{L_{2}} \boldsymbol{g}=\left.u^{*}\right|_{t=0}
$$

Despite its simplicity, this is often not an optimal choice, as it may result in poor scaling of the corresponding discrete optimization problem. For reasons explained hereafter (see also $[15,18]$ ), in many cases it is beneficial to extract gradients in Sobolev spaces, for instance in the space $H^{1(l)}(0,2 \pi)$ equipped with the inner product

$$
\left(z_{1}, z_{2}\right)_{H^{1(l)}}=\frac{1}{\left(1+l^{2}\right)} \int_{0}^{2 \pi}\left[z_{1} z_{2}+l^{2}\left(\partial_{x} z_{1}\right)\left(\partial_{x} z_{2}\right)\right] d x
$$

where $l$ is an adjustable length-scale parameter (in contrast, the space $L_{2}(0,2 \pi)$ does not possess any adjustable parameters). Identification $\mathcal{I}^{\prime}\left(\phi ; \phi^{\prime}\right)=\left(\nabla^{H^{1(l)}} \mathcal{g}, \phi^{\prime}\right)_{H^{1(l)}}$ [cf. (5)] yields, after integration by parts, the gradient $\nabla^{H^{1(l)}} \mathcal{g}$ defined as the solu- 
tion of the following boundary value problem for the Helmholtz equation

$$
\left\{\begin{array}{l}
\frac{1}{1+l^{2}}\left[1-l^{2} \partial_{x}^{2}\right] \nabla^{H^{1(l)}} \mathcal{J}=\left.\bar{u}^{*}\right|_{t=0}, \quad x \in \Omega \\
\nabla^{H^{1(l)}} g(0)=\nabla^{H^{1(l)}} J(2 \pi) .
\end{array}\right.
$$

Thus, the Sobolev space gradient $\nabla^{H^{1(l)}} \mathcal{g}$ is obtained by applying the inverse Helmholtz operator to the "classical" $L_{2}$ gradient. Interestingly, when regarded in Fourier space, the inverse Helmholtz operator is equivalent to a low-pass filter with the cut-off given by the inverse of the length-scale $l$ parameterizing inner product (16). Consequently, extracting gradients in the Sobolev space with the inner product given by (16) has the effect of de-emphasizing components with characteristic length-scales smaller than $l$. As was shown in [18], adjusting this length-scale in the course of the iterative solution of an optimization problem can accelerate convergence of iterations. For example, starting with $l$ large and then gradually decreasing it to zero results in a multiscale procedure targeting first the large-scale structures and then gradually homing in on the smaller scale components of the solution $\tilde{\phi}_{\text {opt }}$ (we note that $\lim _{l \rightarrow 0} \nabla^{H^{1(l)}} \mathcal{g}=\nabla^{L_{2}} \mathcal{g}$ ). This technique can thus be regarded as a combination of operator preconditioning with the variable metric approach mentioned in Introduction. Finally, we emphasize that due to the inclusion $H^{1(l)}(0,2 \pi) \subset L_{2}(0,2 \pi)[25], \nabla^{H^{1(l)}} \mathcal{g}$ does represent an acceptable descent direction for problem $(6)$ as regards smoothness.

In the present work the state and adjoint systems are both solved in the wellresolved setting (on 512 grid points) using a dealiased pseudospectral FourierGalerkin method. Time advancement is performed using an RK3 scheme on the nonlinear term in (6) and the term $u \partial_{x} u^{*}$ in (13), and the $\theta$ method with $\theta=5 / 8$ on the linear terms (see [26]).

\subsection{State Reconstruction for the Navier-Stokes Equation}

As our second model we consider a viscous incompressible flow in a channel $\Omega \triangleq$ $\left(0, L_{1}\right) \times(-1,1) \times\left(0, L_{3}\right)$ periodic in the streamwise and spanwise directions $x_{1}$ and $x_{3}$, and bounded in the direction $x_{2}$ (Fig. 2a). The quantities defined at the wall, i.e., for $x_{2}= \pm 1$, will be distinguished by the subscript " $w$ ". Defining the state vector as $\mathbf{q}=\left[\begin{array}{l}\mathbf{u} \\ p\end{array}\right]$, where $\mathbf{u}=\left[\begin{array}{lll}u_{1} & u_{2} & u_{3}\end{array}\right]^{T}$ is the velocity vector field and $p$ is the 


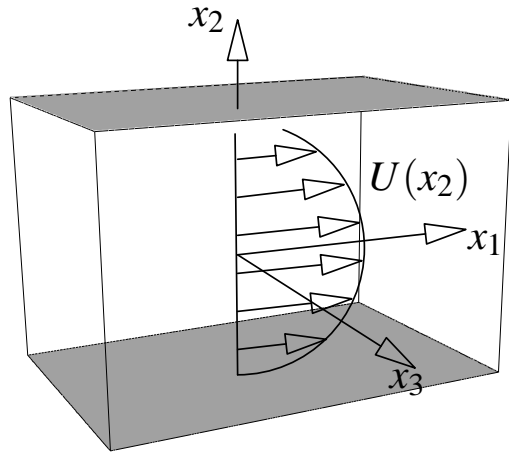

(a)

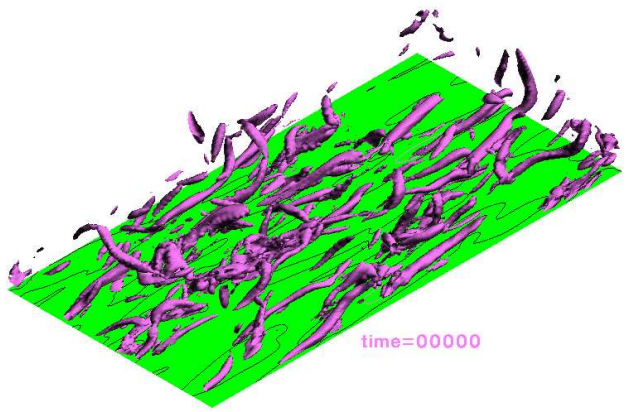

(b)

Fig. 2. Turbulent flow in a channel at $R e_{\tau}=100$ : (a) configuration and (b) visualization of near-wall coherent structures (courtesy of T. Bewley).

pressure field, the system evolution is governed by the Navier-Stokes system

$$
\begin{array}{ll}
\mathcal{N}(\mathbf{q})=\left[\begin{array}{c}
\frac{\partial \mathbf{u}}{\partial t}+(\mathbf{u} \cdot \nabla) \mathbf{u}+\nabla p-v \Delta \mathbf{u} \\
\nabla \cdot \mathbf{u}
\end{array}\right]=\left[\begin{array}{c}
P_{x} \mathbf{i} \\
0
\end{array}\right] & \text { in } \Omega \times(0, T] ; \\
\mathbf{u}=\Phi & \text { at } t=0 ; \\
\left.\mathbf{u}\right|_{w}=0 & \text { on } \partial \Omega,
\end{array}
$$

where $P_{x}$ is the pressure gradient adjusted to maintain a constant mass flux and $\Phi$ is the initial condition. Consistently with the properties of system (18) [24], we will assume that $\Phi \in \mathcal{U}=\mathbf{L}_{2}(\Omega)$. In the present investigation the state $\mathbf{q}$ will be estimated based on information about skin friction and pressure fluctuations at the wall (i.e., for $x= \pm 1$ ) which are a signature of the near-wall coherent structures (Fig. 2b, see also [22] for a discussion concerning the completeness of this set of measurements). We define first a wall measurement vector $\mathbf{m}=\left[m_{1} m_{2} m_{3}\right]^{T}$, where $\left.m_{1} \triangleq \frac{\partial u_{1}}{\partial n}\right|_{w},\left.m_{2} \triangleq p\right|_{w}$, and $\left.m_{3} \triangleq \frac{\partial u_{3}}{\partial n}\right|_{w}$, distributed in time over an assimilation window $[0, T]$ and in space over the channel walls for an "actual" channel-flow system ( $\mathbf{n}$ is defined as an inward-facing normal). Solution of our state estimation problem is obtained as the minimization of a functional $\mathcal{I}(\Phi)$ which represents mathematically the "misfit" of the measurements in the actual and reconstructed systems

$$
\mathcal{J}(\Phi)=\frac{1}{2} \int_{0}^{T}\left[\ell_{1}\left\|\frac{\partial u_{1}}{\partial n}-m_{1}\right\|_{w}^{2}+\ell_{2}\left\|p-m_{2}\right\|_{w}^{2}+\ell_{3}\left\|\frac{\partial u_{3}}{\partial n}-m_{3}\right\|_{w}^{2}\right] d t
$$

where the coefficients $\ell_{1}, \ell_{2}, \ell_{3}$, and the norm $\|\cdot\|_{w}$ are defined appropriately to measure the deviation of the model system from the measurements of the actual flow on the channel walls at $x_{2}= \pm 1$. Note that $\ell_{2}$ is proportional to the square of the (constant) fluid density, $\rho^{2}$, and $\ell_{1}$ and $\ell_{3}$ are proportional to the square of the (constant) fluid viscosity, $\mu^{2}$, in order to make (19) dimensionally consistent. In 
the present work we will consider the case in which $L_{2}$ norms are used such that $\|f\|_{w}^{2} \triangleq \int_{w} f^{2} d S$. The Gâteaux differential of (19) is given by

$\mathcal{J}\left(\Phi ; \Phi^{\prime}\right)=\int_{0}^{T} \int_{w}\left[\ell_{1}\left(\frac{\partial u_{1}}{\partial n}-m_{1}\right) \frac{\partial u_{1}^{\prime}}{\partial n}+\ell_{2}\left(p-m_{2}\right) p^{\prime}+\ell_{3}\left(\frac{\partial u_{3}}{\partial n}-m_{3}\right) \frac{\partial u_{3}^{\prime}}{\partial n}\right] d S d t$

where the equation governing the perturbation vector $\mathbf{q}^{\prime}=\mathbf{q}^{\prime}\left(\Phi ; \Phi^{\prime}\right)=\left[\begin{array}{l}\mathbf{u}^{\prime} \\ p^{\prime}\end{array}\right]$ satisfies the Navier-Stokes system linearized around the state $\mathbf{q}(\Phi)$, i.e.,

$$
\begin{array}{ll}
\mathcal{L}\left(\mathbf{q}^{\prime}\right)=\left[\begin{array}{cl}
\frac{\partial \mathbf{u}^{\prime}}{\partial t}+(\mathbf{u} \cdot \nabla) \mathbf{u}^{\prime}+\left(\mathbf{u}^{\prime} \cdot \nabla\right) \mathbf{u}+\nabla p^{\prime}-v \Delta \mathbf{u}^{\prime} \\
\nabla \cdot \mathbf{u}^{\prime}
\end{array}\right]=\left[\begin{array}{l}
0 \\
0
\end{array}\right] & \text { in } \Omega \times(0, T] ; \\
\mathbf{u}^{\prime}=\Phi^{\prime} & \text { at } t=0 ; \\
\left.\mathbf{u}^{\prime}\right|_{w}=0 & \text { on } \partial \Omega .
\end{array}
$$

Here again the operator $\mathcal{L}: X \rightarrow X^{*}$ is to be understood in the weak sense. Relation (20) can now be transformed to a form consistent with Riesz identity (5) by introducing an adjoint operator $\mathcal{L}^{*}: X \rightarrow X^{*}$ and the corresponding adjoint state $q^{*} \in X$ via the following identity

$$
\left\langle\mathbf{q}^{*}, \mathcal{L} \mathbf{q}^{\prime}\right\rangle_{X \times X^{*}}=\left\langle\mathcal{L}^{*} \mathbf{q}^{*}, \mathbf{q}^{\prime}\right\rangle_{X \times X^{*}}+b
$$

where $\langle\cdot, \cdot\rangle_{X \times X} X^{*}$ represents the duality pairing between the spaces $X$ and its dual $X^{*}$, i.e., given $\mathbf{z}_{1} \in X$ and $\left.\mathbf{z}_{2} \in \mathcal{X}^{*},\left\langle\mathbf{z}_{1}, \mathbf{z}_{2}\right\rangle\right\rangle_{\times X^{*}} \triangleq \int_{0}^{T} \int_{\Omega} \mathbf{z}_{1} \cdot \mathbf{z}_{2} d \Omega d t$. Using integration by parts and the definition of $\mathcal{L}$ in (21), we obtain

$$
\begin{aligned}
& \mathcal{L}^{*} \mathbf{q}^{*}=\left[\begin{array}{c}
-\frac{\partial \mathbf{u}^{*}}{\partial t}-\mathbf{u} \cdot\left[\nabla \mathbf{u}^{*}+\left(\nabla \mathbf{u}^{*}\right)^{T}\right]-\nabla p^{*}-v \Delta \mathbf{u}^{*}, \\
-\nabla \cdot \mathbf{u}^{*}
\end{array}\right], \\
& b=\left.\int_{\Omega}\left(u_{j}^{*} u_{j}^{\prime}\right)\right|_{t=0} ^{t=T} d \Omega-\int_{0}^{T} \int_{w} n_{j}\left[p^{*} u_{j}^{\prime}+u_{j}^{*} p^{\prime}+u_{i}^{*}\left(u_{j} u_{i}^{\prime}+u_{j}^{\prime} u_{i}\right)-v\left(u_{i}^{*} \frac{\partial u_{i}^{\prime}}{\partial x_{j}}-u_{i}^{\prime} \frac{\partial u_{i}^{*}}{\partial x_{j}}\right)\right] d \Omega d t .
\end{aligned}
$$

Defining the adjoint system as

$$
\begin{array}{ll}
\mathcal{L}^{*} \mathbf{q}^{*}=0 & \text { in } \Omega \times[0, T) \\
\mathbf{u}^{*}=0 & \text { at } t=T \\
\left.\begin{array}{l}
u_{1}^{*}\left(x_{1}, \pm 1, x_{3}\right)=\ell_{1} \frac{1}{v}\left(\frac{\partial u_{1}}{\partial n}-m_{1}\right), \\
u_{2}^{*}\left(x_{1}, \pm 1, x_{3}\right)=\ell_{2} \bar{n}_{2}\left(p-m_{2}\right), \\
u_{3}^{*}\left(x_{1}, \pm 1, x_{3}\right)=\ell_{3} \frac{1}{v}\left(\frac{\partial u_{3}}{\partial n}-m_{3}\right),
\end{array}\right\} \text { on } \partial \Omega \\
\end{array}
$$


where $\mathbf{q}^{*}=\left[\begin{array}{l}\mathbf{u}^{*} \\ p^{*}\end{array}\right]$, and using (21) and (22) we can now express Gâteaux differential (20) in a desired form consistent with (5)

$$
\mathcal{g}^{\prime}\left(\Phi ; \Phi^{\prime}\right)=\left.\int_{\Omega} \mathbf{u}^{*}\right|_{t=0} \Phi^{\prime} d \Omega
$$

Thus, this differential (i.e., the sensitivity of the cost functional $\mathcal{I}$ with respect to perturbations $\Phi^{\prime}$ of the initial condition) can be expressed using the solution of adjoint system (23). Relationship (24) can now be employed to extract the gradient required in descent optimization algorithm (4). Since $\mathcal{U}=\mathbf{L}_{2}(\Omega)$ is equipped with the inner product $\left(\mathbf{z}_{1}, \mathbf{z}_{2}\right)_{\mathbf{L}_{2}} \triangleq \int_{\Omega} \mathbf{z}_{1} \cdot \mathbf{z}_{2} d \Omega$ for $\mathbf{z}_{1}, \mathbf{z}_{2} \in \mathbf{L}_{2}(\Omega)$, we immediately obtain

$$
\mathcal{I}^{\prime}\left(\Phi ; \Phi^{\prime}\right)=\left.\int_{\Omega} \mathbf{u}^{*}\right|_{t=0} \Phi^{\prime} d \Omega=\left(\nabla^{\mathbf{L}_{2}} \mathcal{g}, \Phi^{\prime}\right)_{\mathbf{L}_{2}} \Longrightarrow \nabla^{\mathbf{L}_{2}} \mathcal{g}=\left.\mathbf{u}^{*}\right|_{t=0}
$$

As already discussed in Section 2.1, identifying $\mathcal{I}^{\prime}\left(\Phi ; \Phi^{\prime}\right)$ with an $\mathbf{H}^{1(l)}$ inner product defined as $\left(\mathbf{z}_{1}, \mathbf{z}_{2}\right)_{\mathbf{H}^{1(l)}}=\frac{1}{\left(1+l^{2}\right)} \int_{\Omega}\left[\left(z_{1}\right)_{i}\left(z_{2}\right)_{i}+l^{2}\left(\partial_{x_{j}}\left(z_{1}\right)_{i}\right)\left(\partial_{x_{j}}\left(z_{2}\right)_{i}\right)\right] d \Omega$ with implied summation for repeated indices, i.e.,

$$
\mathcal{I}^{\prime}\left(\Phi ; \Phi^{\prime}\right)=\left(\nabla^{\mathbf{H}^{1(l)}} \mathcal{J}, \Phi^{\prime}\right)_{\mathbf{H}^{1(l)}}
$$

then integrating by parts and using the boundary conditions $\left.\nabla^{\mathbf{H}^{1(l)}} \mathcal{J}\right|_{w}=\mathbf{0}$, the gradient $\nabla^{\mathbf{H}^{(l)}} \mathrm{g}$ is obtained as the solution of the following boundary value problem for the Helmholtz equation

$$
\left\{\begin{array}{l}
\frac{1}{1+l^{2}}\left[1-l^{2} \Delta\right]\left(\nabla^{\mathbf{H}^{1(l)}} \mathcal{g}\right)=\left.\mathbf{u}^{*}\right|_{t=0}, \quad \text { in } \Omega \\
\left.\nabla^{\mathbf{H}^{1(l)}} \mathcal{g}\right|_{w}=\mathbf{0}, \\
\nabla^{\mathbf{H}^{1(l)}} \mathcal{g} \text { periodic in } x_{1} \text { and } x_{3} .
\end{array}\right.
$$

The length-scale $l$ plays here the same role as discussed in Section 2.1 and the same comments apply as regards variable metric operator preconditioning.

We will study the case with the Reynolds number $R e_{\tau}=100$. Both Navier-Stokes system (18) and adjoint system (23) are solved numerically with a method combining a finite-difference discretization on a nonuniform grid in the wall-normal direction $x_{2}$ with spectral discretization in the periodic directions $x_{1}$ and $x_{3}$. Timeadvancement is carried out applying the RK3 method to the nonlinear terms in (18) and the term $\mathbf{u} \cdot\left[\nabla \mathbf{u}^{*}+\left(\nabla \mathbf{u}^{*}\right)^{T}\right]$ in $(23)$, and the Crank-Nicolson method to the remaining terms. Further details concerning this numerical method together with benchmark computations are presented in [3]. 


\section{Gradient Extraction in Banach Spaces}

In this Section we show how steepest descent directions can be identified in general Banach spaces not equipped with the Hilbert structure. This idea was introduced by Neuberger [15] in the context of numerical solution of direct problems for PDEs. As regards adjoint-based solution of inverse problems for PDEs, similar ideas were discussed in $[27,28]$. We will assume that the Banach space of controls $\mathcal{U}$ is reflexive, i.e., $\mathcal{U}^{* *}=\mathcal{U}$. Fixing $\phi$, the Gâteaux differential $\mathcal{I}^{\prime}(\phi, \cdot)$ can be regarded as a bounded linear functional on $\mathcal{U}$, i.e., $\mathfrak{I}^{\prime}(\phi, \cdot): \mathcal{U} \rightarrow \mathbb{R}$. As such, the Gâteaux differential admits the representation

$$
\mathcal{I}^{\prime}\left(\phi ; \phi^{\prime}\right)=\left\langle\nabla^{\mathcal{U}} \mathcal{g}, \phi^{\prime}\right\rangle_{\mathcal{U}^{*} \times \mathcal{U}}
$$

where $\nabla^{\mathcal{U}} \mathfrak{g} \in \mathcal{U}^{*}$, i.e., the gradient $\nabla^{\mathcal{U}} \mathfrak{g}$ is an element of the dual space $\mathcal{U}^{*}$ and $\langle\cdot, \cdot\rangle_{\mathcal{U}^{*} \times \mathcal{U}}$ denotes the duality pairing of the spaces $\mathcal{U}$ and $\mathcal{U}^{*}$, i.e., for $z_{1} \in \mathcal{U}$

and $z_{2} \in \mathcal{U}^{*},\left\langle z_{1}, z_{2}\right\rangle_{\mathcal{U}^{*} \times \mathcal{U}} \triangleq \int_{\Omega} z 1 z 2 d x$. We emphasize that the Riesz theorem and inner-product representation (5) are not applicable in the present case when $\mathcal{U}$ is not a Hilbert space. In many important situations the dual space $\mathcal{U}^{*}$ is "larger" than the primal space $\mathcal{U}$, i.e., $\mathcal{U} \subseteq \mathcal{U}^{*}$, in which case the gradient $\nabla^{\mathcal{U}} \mathcal{J}$ may not belong to the control space $\mathcal{U}$ and therefore could not be used to update the control in (4). The reason is that, if $\nabla^{\mathcal{U}} \mathfrak{J} \notin \mathcal{U}$, the gradient $\nabla^{\mathcal{U}} \mathfrak{g}$ may not meet the regularity (smoothness), integrability, etc., conditions required for well-posedness of the original problem $\mathcal{G}(\mathbf{v}, \phi)=0$. In the computational setting this may become apparent in the form of small-scale oscillations and/or singularities appearing in the gradient as the numerical resolution is refined. Thus, in a general case an acceptable steepest descent direction, denoted here $\mathcal{D} \mathcal{I}$, may not be identified with the negative gradient and must be therefore determined in a different way. As shown in $[15,27]$, this can be done defining $\mathcal{D} \mathcal{J}$ to be the unit-norm element of the space $\mathcal{U}$ which minimizes expression (28). In other words, we postulate to find $\mathcal{D} \mathfrak{I}$ as the solution of the following constrained minimization problem

$$
\mathcal{D} \mathcal{I}=\operatorname{argmin}_{\|\vartheta\|_{\mathcal{U}}=1}\left\langle\nabla^{\mathcal{U}} \mathfrak{g}, \vartheta\right\rangle_{\mathcal{U}^{*} \times \mathfrak{U}},
$$

which can be transformed to the more convenient unconstrained form

$$
\mathcal{D} \mathcal{I}=\operatorname{argmin}_{\vartheta \in \mathcal{U}}\left[\left\langle\nabla^{\mathcal{U}} \mathcal{J}, \vartheta\right\rangle_{\mathcal{U}^{*} \times \mathcal{U}}+\frac{\mu}{p}\|\vartheta\|_{\mathcal{U}}^{p}\right]=\operatorname{argmin}_{\vartheta \in \mathcal{U}} \mathcal{F}(\vartheta),
$$

where $p$ is a positive integer chosen to make the resulting expression simpler, $\mu$ is the Lagrange multiplier and $\mathcal{F}: \mathcal{U} \rightarrow \mathbb{R}$ is the Lagrange functional. This problem can be solved by examining the first-order optimality conditions for $\mathcal{F}$. Thus, the steepest descent direction $\mathcal{D} \mathcal{I}$ is characterized by the vanishing of the Gâteaux 
differential of (30), i.e.,

$$
\forall_{\vartheta^{\prime} \in \mathcal{U}} \quad \mathcal{F}^{\prime}\left(\mathcal{D} \mathcal{J} ; \vartheta^{\prime}\right)=\left\langle\nabla^{\mathcal{U}} \mathcal{F}(\mathcal{D} \mathcal{J}), \vartheta^{\prime}\right\rangle_{\mathcal{U}^{*} \times \mathfrak{U}}=0
$$

where $\nabla^{\mathcal{U}} \mathcal{F}: \mathcal{U} \rightarrow \mathcal{U}^{*}$ and $\mathcal{F}^{\prime}$ is the Gâteaux differential of the Lagrange functional $\mathcal{F}$. Thus, we obtain

$$
\nabla^{\mathcal{U}} \mathcal{F}(\mathcal{D} \mathcal{J})=0 \text { in } \mathcal{U}^{*}
$$

as an equation characterizing the descent direction $\mathcal{D} \mathcal{J} \in \mathcal{U}$. Depending on the expression for the norm $\|\cdot\|_{\mathcal{u}}$, equation (32) may be of algebraic or differential type. Specific examples will be analyzed in Sections 3.1 and 3.2. We note that the mapping $\nabla^{\mathcal{U}} \mathcal{F}: \mathcal{U} \rightarrow \mathcal{U}^{*}$ is nonlinear, hence determination of the descent direction in a Banach space involves a nonlinear transformation of the adjoint variable. In order to distinguish them from the gradient $\nabla \mathcal{I}$, we will refer to $\mathcal{D} \mathcal{I}$ as the Lebesgue, Besov, etc., descent directions depending on the choice of the function space $\mathcal{U}$. On the other hand, when the control space $\mathcal{U}$ does possess the Hilbert structure, i.e., $\mathcal{U}=\mathcal{V}$, then after setting $p=2$, (31) becomes

$$
\mathcal{D} \mathcal{I}=\operatorname{argmin}_{\vartheta \in \mathcal{V}}\left[\left\langle\nabla^{\mathcal{V}} \mathfrak{g}, \vartheta\right\rangle_{\mathcal{V}^{*} \times \mathcal{V}}+\frac{\mu}{2}(\vartheta, \vartheta)_{\mathcal{V}}\right],
$$

and, using the now applicable Riesz representation (5), optimality condition (31) becomes

$$
\left\langle\nabla^{\mathcal{V}} \mathcal{g}, \vartheta^{\prime}\right\rangle_{\mathcal{V}^{*} \times \mathcal{V}}+\mu\left(\nabla^{\mathcal{V}} \mathfrak{g}, \vartheta^{\prime}\right)_{\mathcal{V}}=\left(\nabla^{\mathcal{V}} \mathcal{F}, \vartheta^{\prime}\right)_{\mathcal{V}}=0
$$

Transforming (34) using integration by parts we obtain expressions such as (17) and (27). Thus, we conclude that gradient extraction in Hilbert spaces is in fact a particular case of the generic procedure developed in this investigation.

Identification of descent directions in Banach spaces has therefore the effect of a nonlinear change of variables in the optimization problem. Hence, given a Gâteaux differential $\mathcal{I}^{\prime}\left(\phi ; \phi^{\prime}\right)$, there is a distinct direction of the steepest descent associated with every reflexive Banach space $\mathcal{U}$ assumed to contain $\phi^{\prime}$ and, if the optimization problem is convex, these descent directions will ultimately lead to the same minimizer, but the length of the path may be different in different spaces. Obviously, now the important question is how these spaces should be chosen in order to yield a small number of iterations in (4). A mathematically rigorous answer can be given in the case of rather simple problems only, such as the one discussed in Appendix A. In the case of more realistic problems, such as the ones introduced in Sections 2.1 and 2.2, to the author's best knowledge mathematically precise results are unavailable and good choices of the spaces can only be made by way of numerical experimentation. In this sense the proposed approach can be regarded as heuristic. However, from the point of view of many practical optimization problems this is acceptable, because such optimization problems are often solved repeatedly using 
different input data. This is the case, for example, of the variational data assimilation performed on regular basis in order to provide initial conditions for the ensuing weather forecasts (in fact, our model PDE optimization problems from Sections 2.1 and 2.2 were devised to mimic 4DVAR).

As regards identification of descent directions, we will consider families of Banach spaces parametrized by one, or more, continuous parameters, such as, for example, an integrability or differentiability index. In the spirit of the variable metric method, during iterations we will gradually modify the Banach space in which the descent directions are identified by changing values of these parameters. In order to ensure that the underlying PDE problem remains well-posed at every iteration, it is necessary that all of these spaces be contained in $\mathcal{U}$. Thus, we will begin the iterations by first constraining the iterates to some "small" subspace $\mathcal{U}^{(0)}$ of $\mathcal{U}$ which will then "grow" until it becomes numerically indistinguishable from $\mathcal{U}$. Thus, if e.g., $\mathcal{U}=L_{2}$, the intermediate spaces $\mathcal{U}^{(n)}$ must ultimately approach the space $L_{2}$. The family of spaces used for construction of the descent directions can thus be ordered as follows

$$
\mathcal{U}^{(0)} \subseteq \mathcal{U}^{(1)} \subseteq \cdots \subseteq \mathcal{U}^{(n)} \subseteq \cdots \subseteq \mathcal{U}
$$

where $\mathcal{U}^{(n)}$ is the Banach space used at the $n$-th iteration. In general, the problem of finding a continuous family of function spaces linking two given function spaces is the focus of an area of the functional analysis known as the interpolation theory $[25,29]$. There are many profound results in this area, however for our purposes here we will only use some fundamental facts concerning the families of the Lebesgue, Sobolev and Besov spaces. In the remaining parts of this Section we will show how the steepest descent directions for the model problems from Sections 2.1 and 2.2 can be obtained in the Lebesgue and Besov spaces. We omit the case of general Sobolev spaces, because it ultimately produced results similar to the other two cases. The mathematical theory concerning determination of descent directions in general Sobolev spaces was discussed in [15]. We only mention here that the corresponding descent directions are determined by equations formally similar to (17), but with the Laplace operator replaced with the nonlinear $p$-Laplacian.

\subsection{Identification of the Steepest Descent Directions in the Lebesgue Spaces $L_{q}(\Omega)$}

\subsubsection{Optimization Problem for the Kuramoto-Sivashinsky System}

In this Section we characterize the directions of the steepest descent in the space $L_{q}(\Omega)$, characterized by one free parameter $q \in(1, \infty)$, for the optimization problem introduced in Section 2.1. The norm in $L_{q}(\Omega)$ is given by

$$
\|u\|_{L_{q}}=\left(\int_{0}^{2 \pi}|u|^{p} d x\right)^{1 / p}
$$


and the restriction on the index $q$ is related to the requirement of reflexivity, i.e., $\left[L_{q}(\Omega)\right]^{* *}=L_{q}(\Omega)$, which does not hold for $q=1$ and $q=\infty$ [25]. We also note that for $1<q<\infty$ expressions for the norms in $L_{q}(\Omega)$ are differentiable. Furthermore, since $L_{q_{1}}(\Omega) \subset L_{q_{2}}(\Omega)$ for $q_{1}>q_{2}$, the family of spaces $L_{q}(\Omega)$ with the index $q$ decreasing from some $q_{0}$ to 2 forms a hierarchy of the type (35) where $\mathcal{U}=L_{2}$. Following the procedure outlined at the beginning of this Section, we determine the steepest descent direction in the space $L_{q}(\Omega)$ as the solution of the minimization problem

$$
\mathcal{D}^{L_{q}} \mathcal{J}=\operatorname{argmin}_{\|\vartheta\|_{L_{q}}=1}\langle\nabla \mathcal{J}, \vartheta\rangle_{L_{q^{*} \times L_{q}}},
$$

where $L_{q^{*}}(\Omega)=\left[L_{q}(\Omega)\right]^{*}$ with $\frac{1}{q^{*}}+\frac{1}{q}=1$ is the dual space with respect to $L_{q}(\Omega)$. Introducing the Lagrange multiplier $\mu$ and converting (37) to the corresponding unconstrained formulation we obtain

$$
\begin{aligned}
\mathcal{D}^{L_{q}} \mathcal{J}= & \operatorname{argmin}_{\vartheta}\left[\langle\nabla \mathcal{J}, \vartheta\rangle_{L_{q^{*} \times L_{q}}}+\frac{\mu}{p}\|\vartheta\|_{L_{q}}^{p}\right] \\
& =\operatorname{argmin}_{\vartheta} \int_{0}^{2 \pi}\left(\left.u^{*}\right|_{t=0} \vartheta+\frac{\mu}{q}|\vartheta|^{q}\right) d x
\end{aligned}
$$

The first-order optimality condition for (38) yields

$$
\forall_{\vartheta^{\prime} \in L_{q}(\Omega)} \quad \int_{0}^{2 \pi}\left(\left.u^{*}\right|_{t=0}+\mu \mathcal{D}^{L_{q}} \mathcal{g}\left|\mathcal{D}^{L_{q}} \mathcal{g}\right|^{q-2}\right) \vartheta^{\prime} d x=0
$$

which, given the arbitrariness of $\vartheta^{\prime}$, is equivalent to the relationship

$$
\mathcal{D}^{L_{q}} \mathcal{g}\left|\mathcal{D}^{L_{q}} \mathcal{g}\right|^{q-2}=-\left.\frac{1}{\mu} u^{*}\right|_{t=0} \quad \text { in } \Omega
$$

Thus, the steepest descent direction in $L_{q}(\Omega)$ is

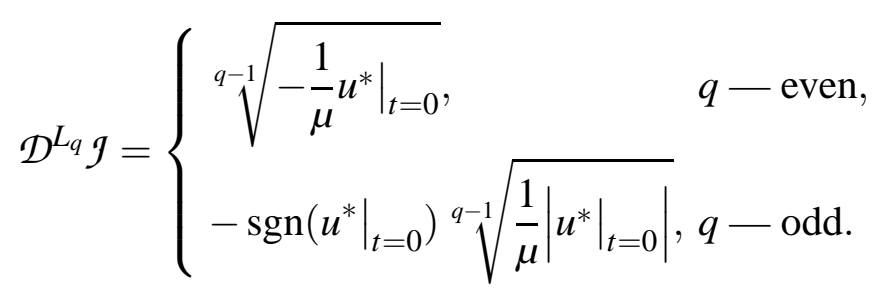

We reiterate that when $q \neq 2$ the steepest descent direction in $L_{p}(\Omega)$ is obtained by applying a nonlinear transformation to the adjoint field $\left.u^{*}\right|_{t=0}$. In the special case $q=2$ we immediately obtain

$$
\mathcal{D}^{L_{2}} \mathfrak{J}=-\left.\frac{1}{\mu} v^{*}\right|_{t=0}=\nabla^{L_{2}} \mathfrak{g}
$$

which coincides with the "classical" Hilbert space expression obtained in Section 2.1. We emphasize that a Lebesgue descent direction $\mathcal{D}^{L_{q}} \mathcal{I}$ can be computed by evaluating algebraic expressions (41) at every point in the domain $\Omega$ and there is 
no need to solve a system of equations. As regards the constant $\mu$, which serves as the Lagrange multiplier in the unconstrained formulation (38), it is chosen to normalize $\mathcal{D}^{L_{q}} \mathfrak{g}$ to unit norm, i.e., $\left\|\mathcal{D}^{L_{q}} \mathfrak{g}\right\|_{L_{q}}=1$. Finally, we comment that in the non-reflexive cases $(q=1$ and $q=\infty)$ the above procedure does not yield meaningful results.

\subsubsection{Optimization Problem for the Navier-Stokes System}

In this Section we characterize the direction of the steepest descent in the space $\mathbf{L}_{q}(\Omega)$, characterized by one free parameter $q \in(1, \infty)$, for the optimization problem introduced in Section 2.2. The $\mathbf{L}_{q}(\Omega)$ norm of a vector-valued field $\mathbf{u}: \Omega \rightarrow \mathbb{R}^{3}$ is given by

$$
\|\mathbf{u}\|_{\mathbf{L}_{q}}=\left(\int_{\Omega}|\mathbf{u}|^{q} d \Omega\right)^{1 / q}
$$

where $|\mathbf{u}|=\sqrt{u_{1}^{2}+u_{2}^{2}+u_{3}^{2}}$. Comparing with the case considered in Section 3.1.1 now the situation is more complicated, because the direction of the steepest descent is a vector field which must satisfy the additional condition of incompressibility. Following the procedure outlined at the beginning of this Section, we determine the steepest descent direction in the space $\mathbf{L}_{q}(\Omega)$ as the solution of the minimization problem

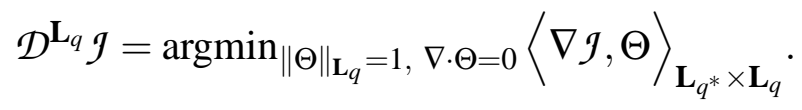

Introducing two Lagrange multipliers $\mu$ and $\eta$, and converting (44) to the corresponding unconstrained formulation we obtain

$$
\begin{aligned}
\mathcal{D}^{\mathbf{L}_{q}} \mathcal{g}= & \operatorname{argmin}_{\Theta}\left[\langle\nabla \mathcal{g}, \Theta\rangle_{\mathbf{L}_{q^{*} \times \mathbf{L}_{q}}}+\frac{\mu}{p}\|\Theta\|_{\mathbf{L}_{q}}^{p}+\int_{\Omega} \eta(\nabla \cdot \Theta) d \Omega\right] \\
& =\operatorname{argmin}_{\Theta} \int_{\Omega}\left(\left.\mathbf{u}^{*}\right|_{t=0} \cdot \Theta+\frac{\mu}{q}|\Theta|^{q}-\Theta \cdot \nabla \eta\right) d \Omega,
\end{aligned}
$$

where we used integration by parts to transform the divergence term and chose the boundary condition for the Lagrange multiplier as $\left.\frac{\partial \eta}{\partial n}\right|_{w}=0$, which annihilated the boundary term arising from this transformation. The first-order optimality condition for (45) yields

$$
\forall_{\Theta^{\prime} \in \mathbf{L}_{q}(\Omega)} \quad \int_{\Omega}\left(\left.\mathbf{u}^{*}\right|_{t=0}+\mu \mathcal{D}^{\mathbf{L}_{q}} \mathcal{g}\left|\mathcal{D}^{\mathbf{L}_{q}} \boldsymbol{g}\right|^{q-2}-\nabla \eta\right) \cdot \Theta^{\prime} d \Omega=0
$$


which, given the arbitrariness of $\Theta^{\prime}$, is equivalent to the following algebro-differential system

$$
\begin{cases}D^{\mathbf{L}_{q}} \mathcal{g}\left|\mathcal{D}^{\mathbf{L}_{q}} \boldsymbol{g}\right|^{q-2}=-\left.\frac{1}{\mu} \mathbf{u}^{*}\right|_{t=0}+\nabla \eta, & \mathbf{x} \in \Omega \\ \Delta \eta=\mu\left(\nabla\left|\mathcal{D}^{\mathbf{L}_{q}} \mathcal{g}\right|^{q-2}\right) \cdot \mathcal{D}^{\mathbf{L}_{q}} \mathcal{g}, & \mathbf{x} \in \Omega \\ \left.\frac{\partial \eta}{\partial n}\right|_{w}=0, & \end{cases}
$$

where the second (Poisson) equation is obtained by applying the divergence operator to the first equation and then using the incompressibility condition $\nabla \cdot\left(\mathcal{D}^{\mathbf{L}_{q}} \mathcal{g}\right)=$ 0 . We note that in the special case $q=2$ we have $\eta \equiv 0$ and (47) reduces to $\nabla^{\mathbf{L}_{2}} \boldsymbol{g}=-\left.\frac{1}{\mu} \mathbf{u}^{*}\right|_{t=0}$ [cf. (25)]. As before, the Lagrange multiplier $\mu$ is adjusted in order to normalize $\mathcal{D}^{\mathbf{L}_{q}} \mathcal{g}$ to the unit $\mathbf{L}_{q}(\Omega)$ norm, i.e., $\left\|\mathcal{D}^{\mathbf{L}_{q}} \mathfrak{g}\right\|_{\mathbf{L}_{q}}=1$. We remark that the procedure described above is closely related to the Helmholtz-Weyl decomposition which is a generalization of the Helmholtz-Hodge decomposition to the spaces $\mathbf{L}_{q}(\Omega)$ [30]. We emphasize that, in contrast to the 1D case where the incompressibility condition was not present [cf. (41)], new problem (47) is nonlocal and therefore after discretization yields a fully coupled nonlinear system. We solve this system using an iterative splitting method which combines Newton's method with globalization [31] applied to the first equation (with $\eta$ frozen during an iteration) with a standard Poisson solver applied to the second equation in (47). For modest values of $q$ convergence usually occurs within a few dozens of iterations.

\subsection{Identification of the Steepest Descent Directions in the Besov Spaces $B_{p, q}^{s}(\Omega)$}

In this Section we characterize the steepest descent directions in the Besov space $B_{p, q}^{s}(\Omega)$ characterized by three adjustable parameters: $s, p$ and $q$. We will consider the parameter ranges $s \geq 0$ and $p, q \geq 2$, for which we have $B_{p, q}^{s}(\Omega) \subset L_{2}(\Omega)$ [25], so that such Besov spaces can form a hierarchy such as (35) with $\mathcal{U}=L_{2}(\Omega)$. Besov spaces arise as a result of interpolation between Sobolev spaces with different integer smoothness, therefore they appear to be good candidates for the variable metric approach developed in this investigation. We emphasize that, since the spaces $L_{q}(\Omega)$ and $B_{q, q}^{0}$ are not equivalent, the Lebesgue descent directions are not special cases of the Besov descent directions. In order to avoid difficulties related to the additional condition of incompressibility in the case of vector fields, we derive the steepest descent directions only for the optimization problem introduced in Section 2.1. From the computational point of view, the most convenient expression for the norm of an element of a Besov space is given in terms of a wavelet decomposition of that element. Defining an orthogonal wavelet $\psi$ such that if we set $\psi_{k, j}(x) \triangleq 2^{\frac{k}{2}} \psi\left(2^{k} x-j\right)$ to be the scaled (by $\left.2^{\frac{k}{2}}\right)$ and translated (by $j 2^{-k}$ ) dilates (by $2^{k}$ ) of the original mother wavelet $\psi$, then $\left\{\psi_{k, j}\right\}_{k, j \in \mathbb{Z}}$ forms an orthogonal basis for $L_{2}(\mathbb{R})$. In an analogous manner we define the scaling function $\varphi$ associated with 
$\psi$ yielding the functions $\varphi_{k, j}(x) \triangleq 2^{\frac{k}{2}} \varphi\left(2^{k} x-j\right)$, such that the set $\left\{\varphi_{k, j}\right\}_{k, j \in \mathbb{Z}}$ is for a fixed $k$ orthonormal in $L_{2}(\mathbb{R})$. When working on a periodic domain $\Omega$, rather than in $\mathbb{R}$, we need to consider suitably periodized wavelets and scaling functions with nonnegative scales $k \geq 0$ only and shifts $j$ defined such that $\operatorname{supp} \psi_{k, j} \cap \Omega \neq \emptyset$. For a specific value of $k$ we will assume allowable shifts $j \in\left\{0, \ldots, N_{k}\right\}$ with $N_{k}$ depending on the particular wavelet used. We will also assume that when $k=0$, the only shift possible is $j=0$. Defining the expansion coefficients as

$$
\begin{aligned}
\alpha_{0} & =\int_{0}^{2 \pi} z(x) \varphi_{0,0}(x) d x \\
\beta_{k, j} & =\int_{0}^{2 \pi} z(x) \psi_{k, j}(x) d x, \quad k=0,1, \ldots, j=0, \ldots, N_{k}
\end{aligned}
$$

a function $z \in L_{2}(0,2 \pi)$ can be represented as

$$
z=\alpha_{0} \varphi_{0,0}+\sum_{k=0}^{\infty} \sum_{j=0}^{N_{k}} \beta_{k, j} \psi_{k, j}
$$

For further details concerning wavelets and the multiresolution analysis we refer the reader to the monographs [32,33]. With these definitions an (equivalent) norm in the Besov space $B_{p, q}^{s}$ can be expressed as [25]

$$
\|z\|_{B_{p, q}^{s}}^{q}=\left|\alpha_{0}\right|^{q}+\sum_{k=0}^{\infty}\left[2^{k p(s+1 / 2-1 / p)} \sum_{j=1}^{N_{k}}\left|\beta_{k, j}\right|^{p}\right]^{\frac{q}{p}} .
$$

Roughly speaking, functions in the space $B_{p, q}^{s}(\Omega)$ have $s$ derivatives in $L_{p}(\Omega)$ with the additional parameter $q$ providing a finer gradation in smoothness. Following the procedure outlined at the beginning of this Section, we determine the steepest descent direction in the space $B_{p, q}^{s}(\Omega)$ as the solution of the minimization problem

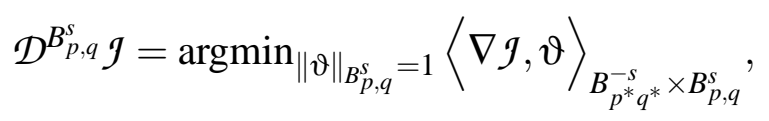

where $B_{p^{*} q^{*}}^{-s}=\left[B_{p, q}^{s}\right]^{*}$ with $\frac{1}{p^{*}}+\frac{1}{p}=1$ is the dual space with respect to $B_{p, q}^{s}$. Introducing the Lagrange multiplier $\mu$ and converting (51) to the corresponding unconstrained formulation we obtain

$$
\begin{aligned}
& \mathcal{D}^{B_{p, q}^{s} \mathcal{I}=} \operatorname{argmin}_{\vartheta}\left[\langle\nabla \mathcal{g}, \vartheta\rangle_{B_{p^{*} q^{*}}^{-s} \times B_{p, q}^{s}}+\frac{\mu}{q}\|\vartheta\|_{B_{p, q}^{s}}^{q}\right] \\
&=\operatorname{argmin}_{\vartheta}\left[\sigma_{0} \alpha_{0}+\sum_{k=0}^{\infty} \sum_{j=0}^{N_{k}} \gamma_{k, j} \beta_{k, j}\right. \\
&+\frac{\mu}{q}\left\{\left(\left|\alpha_{0}\right|^{q}+\sum_{k=0}^{\infty}\left[2^{k p(s+1 / 2-1 / p)} \sum_{j=0}^{N_{k}}\left|\beta_{k, j}\right|^{p}\right]^{\frac{q}{p}}\right\}\right],
\end{aligned}
$$


where the following wavelet representations were employed for the gradient $\nabla \mathfrak{g}=$ $\sigma_{0} \varphi_{0,0}+\sum_{k=0}^{\infty} \sum_{j=0}^{N_{k}} \gamma_{k, j} \psi_{k, j}$ and the element $\vartheta=\alpha_{0} \varphi_{0,0}+\sum_{k=0}^{\infty} \sum_{j=0}^{N_{k}} \beta_{k, j} \psi_{k, j}$. The first-order optimality condition for (52) yields

$$
\begin{aligned}
& \forall_{\alpha_{0}^{\prime} \in \mathbb{R}, \quad \beta_{k, j}^{\prime} \in l_{p}\left(l_{q}\right)} \quad \sum_{j}\left[\sigma_{0}+\mu\left|\alpha_{0}\right|^{q-2} \alpha_{0}\right] \alpha_{0}^{\prime} \\
& +\sum_{k=0}^{\infty} \sum_{j=0}^{N_{k}}\left[\gamma_{k, j}+\mu q 2^{k p(s+1 / 2-1 / p)}\left(\sum_{l}\left|\beta_{k, l}\right|^{p}\right)^{\frac{q}{p}-1}\left|\beta_{k, j}\right|^{p-2} \beta_{k, j}\right] \beta_{k, j}^{\prime}=0,
\end{aligned}
$$

where $\alpha_{0}^{\prime}$ and $\left\{\beta_{k, j}^{\prime}\right\}_{k, j \in \mathbb{Z}}$ are the wavelet expansion coefficients of the perturbation element $\vartheta^{\prime}$. Given the arbitrariness of $\vartheta^{\prime},(53)$ is equivalent to the following infinite system of algebraic equations

$$
\begin{array}{ll}
\mu\left|\alpha_{0}\right|^{q-2} \alpha_{0}=-\sigma_{0}, & j=1, \ldots, N_{j}, \\
\mu q 2^{k q(s+1 / 2-1 / p)}\left(\sum_{l=0}^{N_{k}}\left|\beta_{k, l}\right|^{p}\right)^{\frac{q}{p}-1}\left|\beta_{k, j}\right|^{p-2} \beta_{k, j}=-\gamma_{k, j}, & k=0, \ldots, \infty .
\end{array}
$$

We note that in the special case $p=q$ system (54b) uncouples. When $p \neq q$ we can solve system (54) by first truncating it at the number of levels consistent with the spatial discretization (here $k_{\max }=\log _{2} 1024=10$ ) and using Newton's method with globalization [31] to solve the resulting nonlinear algebraic system. As a matter of fact, since system (54b) decouples for different values of $k$, Newton's method can be used independently for every value of $k$ which reduces the size of the linear algebraic problems that need to be solved at every iteration and also mitigates attendant problems with conditioning. In our computations reported in Section 4.1 wavelet decompositions were computed using the "symmlet" wavelet with 10 vanishing moments. This was done with the help of the MATLAB toolbox wavelab [34]. For modest values of the difference $p-q$ this approach leads to quite rapid convergence. We also emphasize that, because of the relationships between the Besov norms and wavelet decompositions [cf. (50)], determination of Besov descent directions is in fact equivalent to nonlinear wavelet filtering applied to the adjoint field as defined by (54) (the filtering is nonlinear, because modifications of the wavelet coefficients depend on the coefficients themselves). This is therefore analogous to determination of gradients in the Hilbert-Sobolev spaces $H^{1(l)}(\Omega)$ which can in turn be interpreted as low-pass Fourier filtering applied to the adjoint field (see Section 2.1). While the linear Fourier filtering modifies components based on their wavenumbers only, the wavelet filtering does so based on their relative "coherence" [21]. Finally, we remark that the utility of wavelet decompositions for computation of Besov descent directions was first recognized in the image processing literature $[20,35]$. 


\section{Computational Results}

In this Section we illustrate the utility of the proposed approach by comparing its performance to the standard approach based on the $L_{2}$ and $H^{1}$ gradients. We do this for the two model problems introduced in Sections 2.1 and 2.2. The actual computational complexity of a state estimation problem depends on several factors, such as the quality of the initial guess, length of the assimilation window, importance of nonlinear effects and the rank of the observation operator. In order to exhibit more clearly the potential of the proposed method, in both model problems we will choose these parameters in such a way as to make the state estimation problem quite hard and therefore practically unsolvable using the standard methods, in which case the iterations either get stuck in local minima, or take excessively long to converge. In both cases iterations (4) are carried out using the Polak-Ribiere version of the conjugate gradient (CG) method [7]. The "momentum" term in the CG method is calculated using the standard $L_{2}$ inner product and is reset to zero every 20 iterations. Line minimization of the cost functional along the descent direction is performed at every iteration using Brendt's method [36].

\subsection{Results Concerning State Reconstruction for the Kuramoto-Sivashinsky Equa- tion}

The problem set-up is the same as the problem investigated in [18], i.e., $\Lambda_{r}=$ $\{1, \ldots, 50\}$ in $(8)$ and the initial guess is $\phi^{(0)}=0$, except that now we use a longer assimilation window $[0, T]$ with $T=5 \cdot 10^{-6}$ corresponding to 500 time steps. As our computational experience shows, this is enough to make the optimization problem significantly more difficult than for 300 time steps which was the case studied in [18]. Solution of this state estimation problem was attempted using the Lebesgue and Besov descent directions in addition to the classical $L_{2}$ and $H^{1}$ gradients. Consistently with hierarchy (35), the parameters $l_{n}, s_{n}, p_{n}$ and $q_{n}$ characterizing the spaces $H^{1\left(l_{n}\right)}, L_{q_{n}}$, and $B_{p_{n}, q_{n}}^{s_{n}}$ at the $n$-th iteration are changing according to the expressions

$$
\begin{aligned}
l_{n} & =l_{0} \omega_{l}^{-n}, & & s_{n}=s_{0} \omega_{s}^{-n}, \\
p_{n} & =2+\left(p_{0}-2\right) \omega_{p}^{-n}, & & q_{n}=2+\left(q_{0}-2\right) \omega_{q}^{-n},
\end{aligned}
$$

where $l_{0}, s_{0}, p_{0}$ and $q_{0}$ are the starting values and $\omega_{l}, \omega_{s}, \omega_{p}$ and $\omega_{q}$ are suitably chosen decrease parameters, all greater than unity. We emphasize that with these

choices of the parameters the spaces $H^{1\left(l_{n}\right)}, L_{q_{n}}$, and $B_{p_{n}, q_{n}}^{s_{n}}$ become for $n \rightarrow \infty$ numerically indistinguishable from the space $L_{2}$. Below we summarize the best results obtained with each type of the descent direction after a rather modest amount of computational tests performed to find good values of the parameters $l_{0}, s_{0}, p_{0}$, $q_{0}, \omega_{l}, \omega_{s}, \omega_{p}$ and $\omega_{q}$. These parameter values are collected in Table 1. 


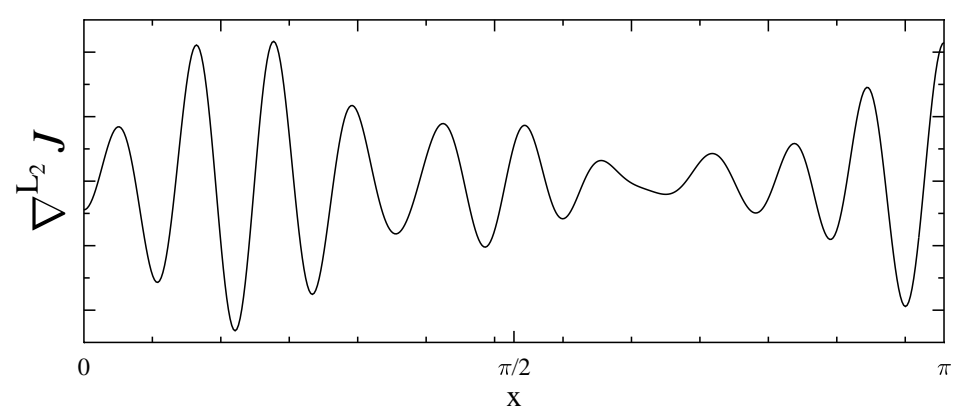

(a)

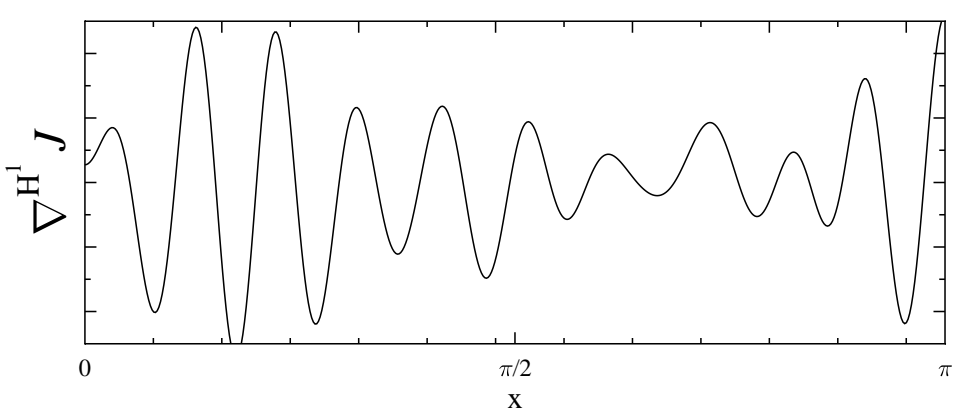

(b)

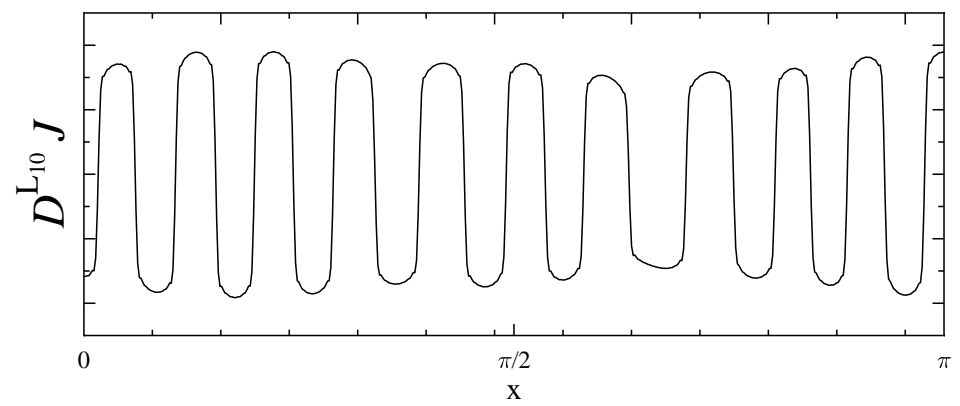

(c)

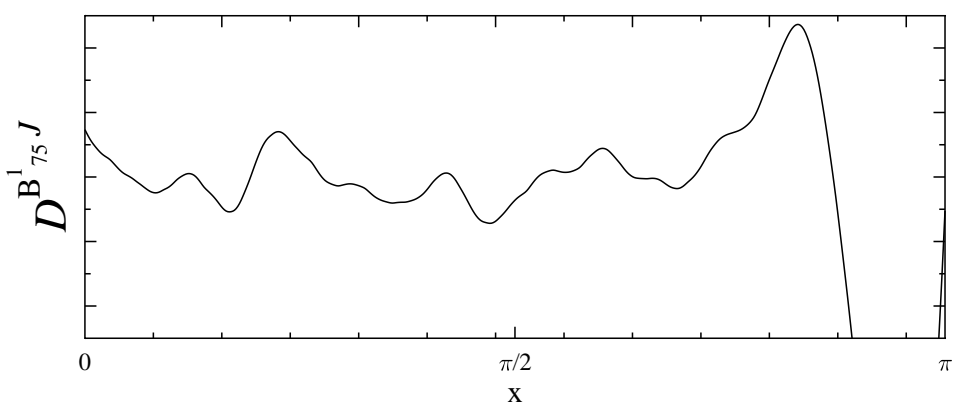

(d)

Fig. 3. Descent directions identified at the first iteration in the spaces (a) $L_{2}(\Omega)$, (b) $H^{1(0.2)}(\Omega)$, (c) $L_{10}(\Omega)$ and (d) $B_{7,5}^{1}(\Omega)$. For the sake of clarity, only half of the domain $\Omega$ is shown. 
Table 1

\begin{tabular}{c|cccccccc}
$\mathrm{CASE}$ & $l_{0}$ & $\omega_{l}$ & $s_{0}$ & $\omega_{s}$ & $p_{0}$ & $\omega_{p}$ & $q_{0}$ & $\omega_{q}$ \\
\hline$L_{2}$ & - & - & - & - & - & - & - & - \\
$H^{1}$ & 0.2 & 1.05 & - & - & - & - & - & - \\
$L_{q}$ & - & - & - & - & - & - & 10 & 2 \\
$B_{p, q}^{s}$ & - & - & 1 & 1.1 & 7 & 1.1 & 5 & 1.1
\end{tabular}

Parameters characterizing the descent directions in different function spaces employed to solve the optimization problem from Section 2.1 [cf. (55)]. The number of non-blank entries in each line is equal to the number of adjustable parameters in the corresponding case.

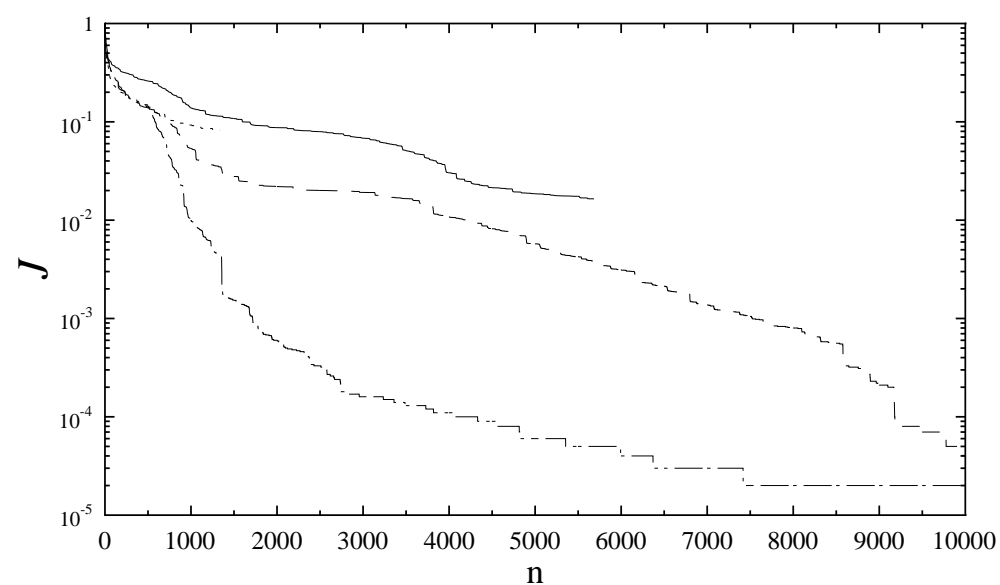

Fig. 4. Decrease of cost functional (7) as a function of the iteration count $n$ in the following cases (cf. Table 1): (solid) $L_{2}$, (dotted) $H^{1}$, (dashed) $L_{q}$ and (dash-dotted) $B_{p, q}^{s}$.

We begin the presentation of the results by showing in Fig. 3 the descent directions obtained at the first iteration in the different cases listed in Table 2.1. We note that while the shape of the $H^{1}$ gradient appears quite close to the shape of the $L_{2}$ gradient, the shapes of the $L_{q}$ and $B_{p, q}^{s}$ descent directions differ from it quite significantly. Next in Fig. 4 we present the histories of the cost functional $\mathcal{I}\left(\tilde{\phi}^{(n)}\right)$ during iterations in the four cases $L_{2}, H^{1}, L_{q}$, and $B_{p, q}^{s}$. We note that the iterates in the cases $L_{2}$ and $H^{1}$ get stuck in local minima. On the other hand, in the cases $L_{q}$ and $B_{p, q}^{s}$ we observe what appears to be convergence to a global minimum, and in the case $B_{p, q}^{s}$ this convergence occurs quite rapidly. These findings are corroborated by the results shown in Fig. 5 where we present distribution of the reconstruction error measures

$$
\mathcal{E}_{X}(t) \triangleq \frac{\left\|\tilde{u}\left(t, \tilde{\phi}^{\left(n_{\max }\right)}\right)-\tilde{u}_{a c t}(t)\right\|_{X}}{\left\|\tilde{u}_{a c t}(t)\right\|_{X}},
$$

defined within the assimilation window $[0, T]$ at the end of the iterations (i.e., for $\left.n=n_{\max }\right)$. The norms $\|\cdot\|_{X}$, where $X=H^{-1}, L_{2}, H^{1}$, are defined using the Parceval 


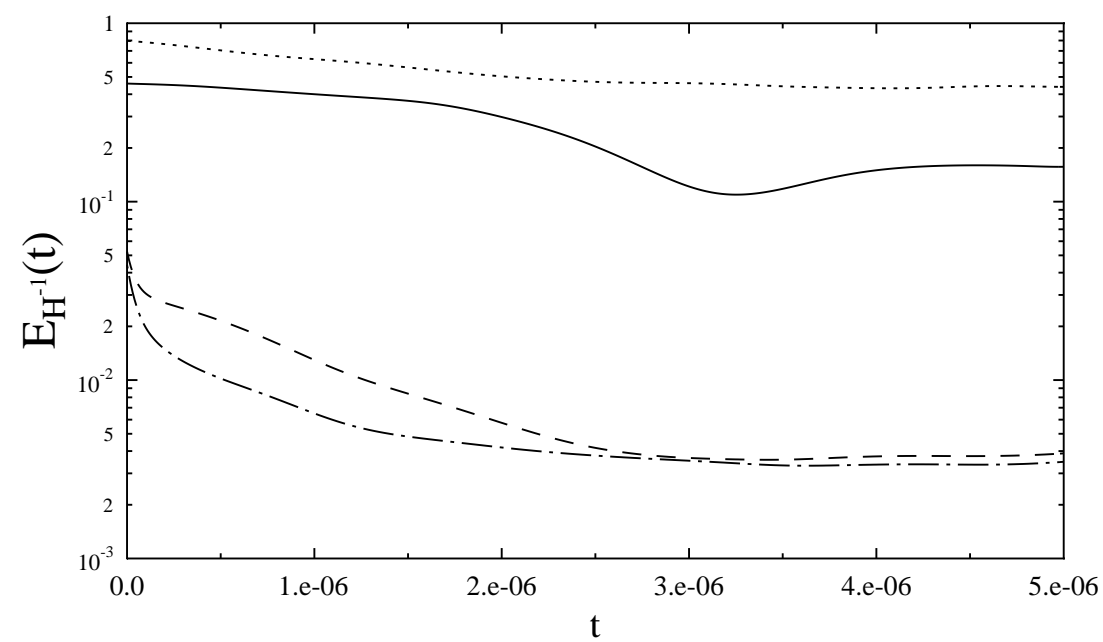

(a)

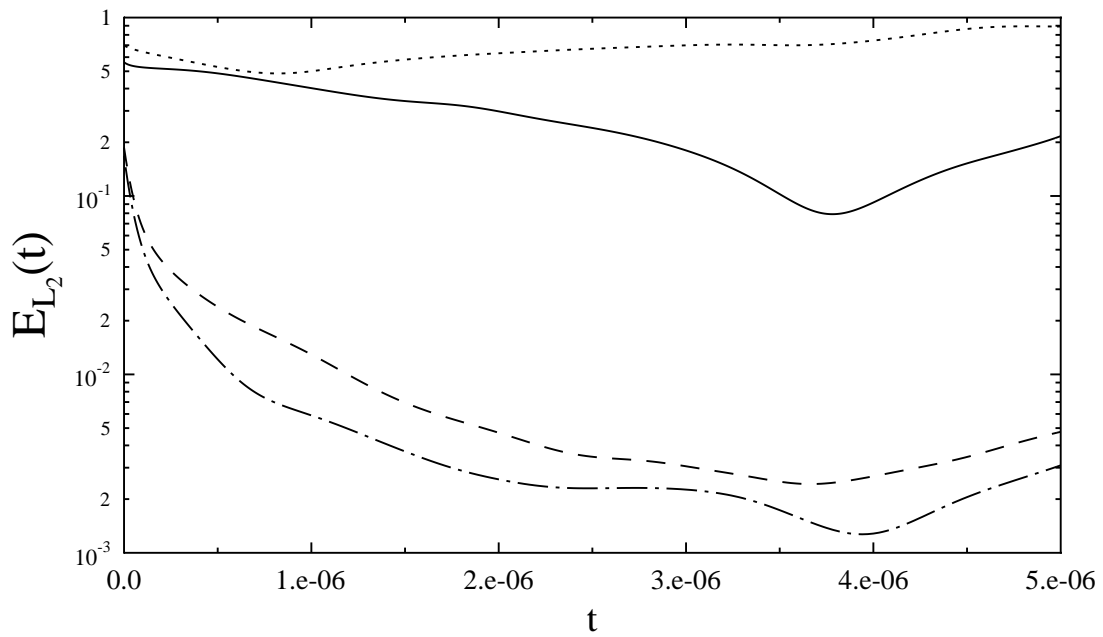

(b)

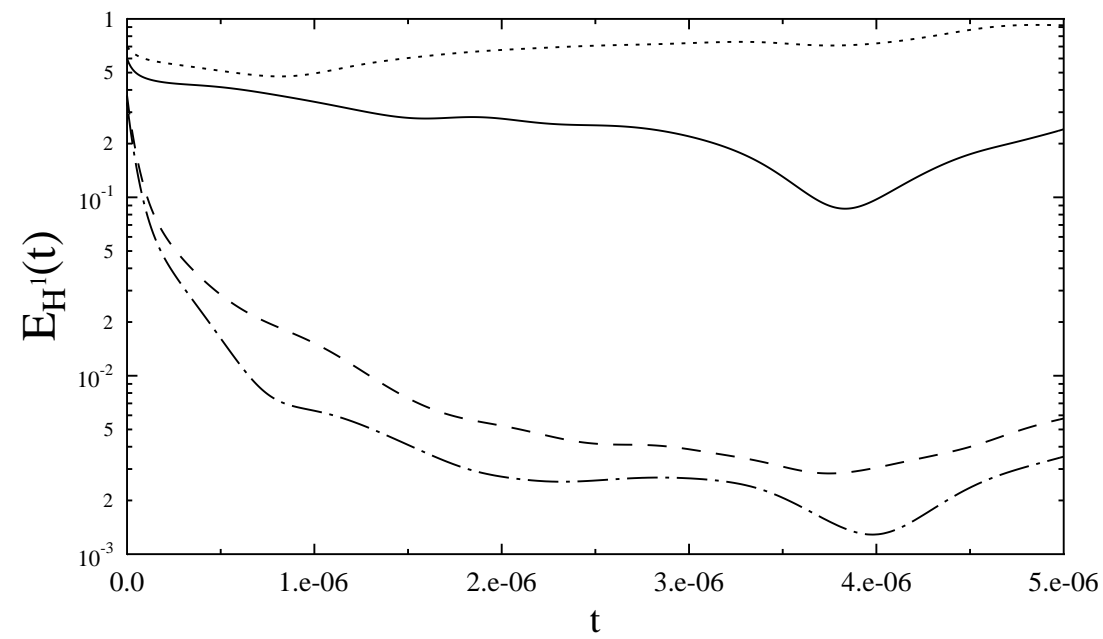

(c)

Fig. 5. Distribution of the reconstruction error measures (a) $\mathcal{E}_{H^{-1}}$, (b) $\mathcal{E}_{L_{2}}$, and (c) $\mathcal{E}_{H^{1}}$ [cf. (56)] within the assimilation window $[0, T]$ at the end of the iterations for the following cases (cf. Table 1): (solid) $L_{2}$, (dotted) $H^{1}$, (dashed) $L_{q}$ and (dash-dotted) $B_{p, q}^{s}$. 
Table 2

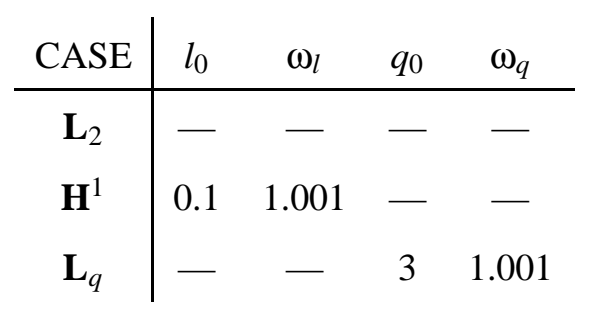

Parameters characterizing the descent directions in different function spaces employed to solve the optimization problem from Section 2.2 [cf. (55)]. The number of non-blank entries in each line is equal to the number of adjustable parameters in the corresponding case.

identity and denoting $\hat{z}_{k}$ the Fourier transform of $z(x)$ as

$$
\|z\|_{H^{-1}}^{2} \triangleq \sum_{k=1}^{\infty} k^{-2}\left|\hat{z}_{k}\right|^{2}, \quad\|z\|_{L_{2}}^{2} \triangleq \sum_{k=0}^{\infty}\left|\hat{z}_{k}\right|^{2}, \quad\|z\|_{H^{1}}^{2} \triangleq \sum_{k=0}^{\infty} k^{2}\left|\hat{z}_{k}\right|^{2} .
$$

These norms are defined here in Fourier space in order to emphasize the fact that (56) measures the accuracy of reconstruction at, respectively, large, intermediate and small scales [18]. We observe that by far the best reconstruction with regard to all three metrics is obtained in the cases, respectively, $B_{p, q}^{s}$ and $L_{q}$. We emphasize that in the $B_{p, q}^{s}$ case a significant reduction of the cost functional was obtained after much fewer iterations. Clearly, the poorest reconstruction was obtained in the case $H^{1}$.

\subsection{Results Concerning State Reconstruction for the Navier-Stokes System}

The problem set-up is the same as in [22], i.e., the initial guess $\Phi^{(0)}$ is taken as the mean flow and the assimilation window $[0, T]$ has the length of 100 viscous time units $t^{+}$corresponding to 330 time steps. As already pointed out in [22], this combination of parameters makes the present state estimation problem very challenging. Solution of this problem is attempted using Lebesgue descent directions introduced in Section 3.1.2, in addition to the classical $\mathbf{H}^{1}$ and $\mathbf{L}_{2}$ gradients. The parameters $l_{n}$ and $q_{n}$ characterizing the spaces $\mathbf{L}_{q_{n}}(\Omega)$ and $\mathbf{H}^{1\left(l_{n}\right)}(\Omega)$ at the $n$-th iteration change according to expressions (55). Below we summarize the best results obtained with each type of the descent direction after a rather modest amount of computational tests performed to find good values of the parameters $l_{0}, q_{0}, \omega_{l}$ and $\omega_{q}$. These parameter values are collected in Table 2 .

We begin the presentation of the results by showing in Fig. 6 the histories of the functional $\mathcal{I}\left(\tilde{\Phi}^{(n)}\right)$ during iterations in the three cases $\mathbf{L}_{2}, \mathbf{H}^{1}$ and $\mathbf{L}_{q}$. We note that, while the decrease of $\mathcal{I}$ is in all cases rather slow, it is clearly the fastest in the $\mathbf{L}_{q}$ case and the slowest in the $\mathbf{L}_{2}$ case. Next in Fig. 7 we present distribution of the error norm

$$
\mathcal{E}_{\mathbf{L}_{2}}(t) \triangleq \frac{\left\|\tilde{\mathbf{v}}\left(t, \tilde{\Phi}^{(500)}\right)-\tilde{\mathbf{v}}_{a c t}(t)\right\|_{\mathbf{L}_{2}}}{\left\|\tilde{\mathbf{v}}_{a c t}(t)\right\|_{\mathbf{L}_{2}}}
$$




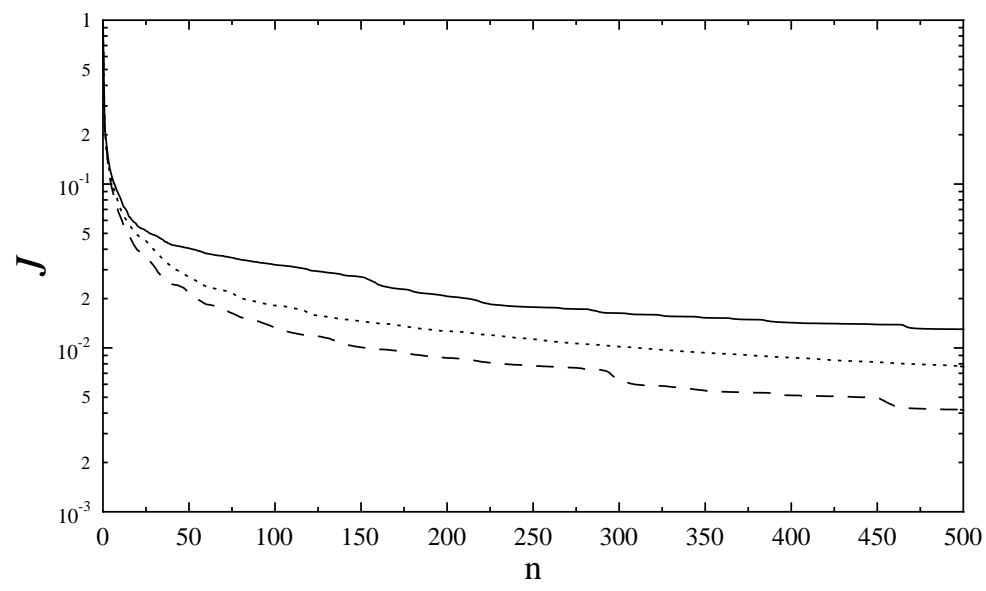

Fig. 6. Decrease of cost functional (19) as a function of the iteration count $n$ in the following cases (cf. Table 2): (solid) $\mathbf{L}_{2}$, (dotted) $\mathbf{H}^{1}$ and (dashed) $\mathbf{L}_{q}$.

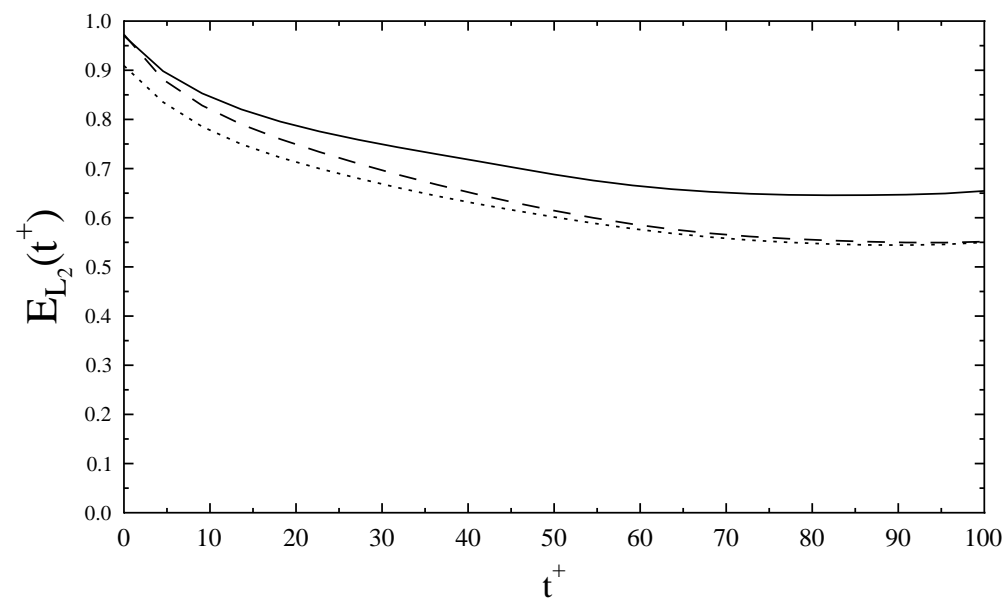

Fig. 7. Distribution of the reconstruction error measure $\mathcal{E}_{\mathbf{L}_{2}}$ [cf. (58)] within the assimilation window $[0, T]$ after $n=500$ iterations in the following cases (cf. Table 2): (solid) $\mathbf{L}_{2}$, (dotted) $\mathbf{H}^{1}$ and (dashed) $\mathbf{L}_{q}$.

within the assimilation window $[0, T]$ after $n=500$ iterations [due to difficulties in computing the $\mathbf{H}^{-1}$ and $\mathbf{H}^{1}$ norms, we present here results for the $\mathbf{L}_{2}$ norm only, cf. (43)]. While in none of the cases can we claim that the original flow has been successfully reconstructed, the progress towards the actual minimizer $\Phi_{a c t}$ is in the cases $\mathbf{H}^{1}$ and $\mathbf{L}_{q}$ significantly better than in the case $\mathbf{L}_{2}$, with the case $\mathbf{H}^{1}$ yielding the lowest errors over the whole assimilation window. We conclude our discussion by mentioning that state estimation in the present channel flow problem with distributed wall measurements was also investigated using other methods, including Taylor-series expansions in [22] and Kalman filtering in [37]. 


\section{Summary and Conclusions}

In this investigation we proposed a method for accelerating convergence of gradientbased optimization of PDE systems. Inspired by the idea of operator preconditioning of Farago and Karátson [14], our approach uses descent directions constructed in continuously varying function spaces as a way of preconditioning the iterations. We show that extraction of descent directions in general Banach spaces not equipped with the Hilbert structure is in fact equivalent to a nonlinear change of the control variables. For nonconvex problems such a change of variables may offer the possibility of "smoothing" the control space more effectively than can be done with a linear change of variables (which is equivalent to merely stretching and contracting the space in the different directions). We showed how such descent directions can be characterized and computed in the Lebesgue and Besov spaces commonly arising in analysis on nonlinear PDEs [25]. These function spaces are parametrized by, respectively, one and three parameters which can be adjusted in order to accelerate convergence. Ideally, a prescription of how this should be done should come from the mathematical analysis of the PDE optimization problem, however, at least for the problems of the type proposed in Sections 2.1 and 2.2, such results are unlikely to be within reach in the foreseeable future. Therefore, these parameters need to be determined by way of computational experimentation. Our results concerning the Kuramoto-Sivashinsky equation show that with suitably chosen Lebesgue and Besov descent directions one can solve optimization problems for which the linear approach with the $L_{2}$ and $H^{1}$ gradients fails. Furthermore, using the Besov descent directions this could be done performing relatively few iterations. The reasons for the superior performance of the Besov descent directions appear related to their larger number of independent free parameters which offer more possibilities for a topology change than are available in the other cases. As regards the Navier-Stokes problem, our results indicate that approaches based on variable Sobolev gradients and Lebesgue descent directions exhibit similar performance which is better than in the standard $\mathbf{L}_{2}$ approach. It is also worth noting that, given the rate of change of the different parameters in the case of both model problems [cf. (55), and Tables 1 and 2], the Lebesgue and Besov spaces used remained significantly different from the space $L_{2}$ during initial iterations only. This initial effect was however important enough to result in much faster convergence observed also at later iterations. Thus, the two sets of results obtained for two different model problems show the usefulness of an additional flexibility in the design of an iterative process offered by the alternative descent directions proposed here. Our computational experience with problems different than the cases reported here indicates that Banach descent directions are particularly useful in hard problems in which the classical gradients cannot provide satisfactory performance. As a matter of fact, computational evidence showing that convergence of iterations in some functional spaces is more rapid than in other possibly might guide the mathematical analysis of such problems, especially as regards finding for them a natural functional space setting. The fact that "optimal" values of these parameters must be found by experimentation is 
not a serious practical limitation, because in actual applications such optimization problems are often solved repeatedly for a given system using different data (e.g., data assimilation in numerical weather prediction [5]). Thus, once determined, the sets of "optimal" parameters can be reused to accelerate optimizations performed subsequently. From the heuristic point of view, our preconditioning approach can be regarded as an application of a nonlinear filter to the $L_{2}$ descent direction. After its parameters are suitably calibrated, this nonlinear filter acts to emphasize components of the descent direction which are important for convergence and deemphasize those which are assumed to represent noise. We should also mention that the computational cost involved in determining a Lebesgue or Besov descent direction is insignificant comparing to the cost of a single iteration. Finally. we remark that using finite-dimensional emulations of the norms used in the different Banach spaces, the present approach can also be easily applied in the context of the "discretize-then-differentiate" methods.

\section{Acknowledgments}

The authors wishes to thank Prof. John Neuberger for helpful discussions and comments. This research was supported by an NSERC Discovery Grant (Canada).

\section{A Operator Preconditioning in Numerical Solution of a Linear Boundary Value Problem}

In this Appendix we use the Ritz method applied to the Poisson equation in a 1D periodic domain $\Omega=(0,2 \pi)$ to illustrate the importance of extracting gradients in suitable spaces. The problem we consider is thus

$$
\left\{\begin{array}{l}
-\Delta w=g, \\
w(0)=w(2 \pi),
\end{array} \quad \Delta: H_{p e r}^{1}(\Omega) \rightarrow H_{p e r}^{-1}(\Omega),\right.
$$

where $w \in \mathcal{U}=H_{\text {per }}^{1}(\Omega)$ and $H_{\text {per }}^{1}(\Omega)$ is the Sobolev space of periodic functions with square integrable first derivatives. One way of solving (A.1) is by finding minimizers of a cost functional $\mathcal{I}: H_{p e r}^{1}(\Omega) \rightarrow \mathbb{R}$ defined as

$$
\mathcal{I}(\vartheta) \triangleq \int_{0}^{2 \pi}\left[\frac{1}{2}(\nabla \vartheta)^{2}-g \vartheta\right] d x
$$

which can be done using iterations (4). Assuming (incorrectly!) that $\mathcal{U}=L_{2}(\Omega)$, the gradient of (A.2) is $\nabla^{L_{2}} \mathcal{J}=-\Delta \vartheta-g$, whereas if one takes $\mathcal{U}=H_{p e r}^{1}(\Omega)$, the gradient is $\nabla^{H^{1}} \mathcal{J}=\Delta^{-1}[-\Delta \vartheta-g]$. 
It is well-known [7], that the rate of convergence of gradient iterations (4) depends on the condition number $\varkappa(\tilde{\Xi})$ of the discrete Hessian $\tilde{\Xi}$ of (A.2). It can be computed noting that $\mathcal{I}^{\prime \prime}\left(\vartheta ; \vartheta^{\prime}, \vartheta^{\prime \prime}\right)=-\int_{0}^{2 \pi} \vartheta^{\prime \prime}\left(\Delta \vartheta^{\prime}\right) d x=-\int_{0}^{2 \pi}\left(\Delta \vartheta^{\prime \prime}\right) \vartheta^{\prime} d x$. Then, assuming again that $\mathcal{U}=L_{2}(\Omega)$, we have

$$
g^{\prime \prime}\left(\vartheta ; \vartheta^{\prime}, \vartheta^{\prime \prime}\right)=\left(\Xi_{L_{2}} \vartheta^{\prime}, \vartheta^{\prime \prime}\right)_{L_{2}} \quad \Rightarrow \quad \Xi_{L_{2}} \vartheta^{\prime}=-\Delta \vartheta^{\prime} \quad \Rightarrow \quad \Xi_{L_{2}}=-\Delta .
$$

Using Fourier harmonics as the basis functions, the Hessian $\Xi_{L_{2}}$ can be represented as an infinite diagonal matrix with entries proportional to the wavenumbers squared $k^{2}$. After truncating the problem at some $k_{\max }$, the discrete Hessian condition number can be expressed as $\varkappa\left(\tilde{\Xi}_{L_{2}}\right)=\left(\frac{k_{\max }}{k_{\min }}\right)^{2}$, which grows without bound as the resolution is refined (i.e., as $k_{\max } \rightarrow \infty$ ). Thus, as the grid is refined, the conditioning of the minimization problem deteriorates rendering it practically unsolvable.

On the other hand, taking $\mathcal{U}=H_{p e r}^{1}(\Omega)$ we have

$$
g^{\prime \prime}\left(\vartheta ; \vartheta^{\prime}, \vartheta^{\prime \prime}\right)=\left(\Xi_{H^{1}} \vartheta^{\prime}, \vartheta^{\prime \prime}\right)_{H^{1}} \quad \Rightarrow \quad \Xi_{H^{1}} \vartheta^{\prime}=\vartheta^{\prime} \quad \Rightarrow \quad \Xi_{H^{1}}=\mathrm{Id}
$$

so that the condition number $\varkappa\left(\tilde{\Xi}_{H^{1}}\right) \equiv 1$ regardless of the numerical resolution used. This is thus an instance of a perfect conditioning and convergence can in principle be achieved in a single iteration. It must be, however, borne in mind that determination of the gradient $\nabla^{H^{1}} \mathcal{J}$ is fact equivalent to solution of problem (A.1) itself. This example contrasting two extreme situations underlines the usual tradeoffs between the effectiveness and ease of computation of preconditioning operators.

\section{References}

[1] B. Mohammadi and O Pironneau, "Applied Shape Optimization for Fluids", Oxford University Press, (2001).

[2] J. R. R. A. Martins, J. J. Alonso and J. J. Reuther, "High-fidelity aerostructural design optimization of a supersonic business jet", Journal of Aircraft 41, 523-530, (2004).

[3] T. R. Bewley, P. Moin and R. Temam, "DNS-based predictive control of turbulence: an optimal benchmark for feedback algorithms", J. Fluid Mech. 447, 179-225, 2001.

[4] B. Protas and A. Styczek, "Optimal Rotary Control of the Cylinder Wake in the Laminar Regime", Physics of Fluids 14(7), 2073-2087, 2002.

[5] E. Kalnay, "Atmospheric Modeling, Data Assimilation and Predictability", Cambridge University Press, (2003).

[6] G. Mathew, I. Mezic, S. Grivopoulos, U. Vaidya and L. Petzold, "Optimal Control of Mixing in Stokes Fluid Flows”, 580, 261-281, (2007). 
[7] J. Nocedal and S. Wright, "Numerical Optimization", Springer, (2002).

[8] J. L. Lions, Contrôle Optimal des Systèmes Gouvernés par des Equations aux Dérivées Partialles, Dunod, Paris (1969), English translation, Springer-Verlag, New-York.

[9] F. Abergel and R. Temam, "On Some Control Problems in Fluid Mechanics", Theoret. Comput Fluid Dyn. 1, 303-325, (1990).

[10] M. D. Gunzburger, "Perspectives in flow control and optimization", SIAM, Philadelphia, (2003).

[11] R. M. Lewis and S. G. Nash, "Model problems for the multigrid optimization of systems governed by differential equations", SIAM Journal on Scientific Computing 26, 1811-1837, (2005).

[12] J. E. Dennis and R. B. Schnabel, "Numerical Methods for Unconstrained Optimization and Nonlinear Equations", Prentice-Hall, (1983).

[13] O. Axelsson, "Iterative Solution Methods", Cambridge University Press, (1994).

[14] I. Farago and J. Karátson, "Numerical Solution of Nonlinear Elliptic Problems Via Preconditioning Operators: Theory and Applications", Nova Science, (2002).

[15] J. Neuberger, "Sobolev Gradients and Differential Equations", Springer, (1997).

[16] M. S. Berger, "Nonlinearity and Functional Analysis", Academic Press, (1977).

[17] S. Sial, J. Neuberger, T. Lookman and A. Saxena, "Energy minimization using Sobolev gradients: application to phase separation and ordering", J. Comp. Phys. 189, 88-97, (2003).

[18] B. Protas, T. Bewley and G. Hagen, "A comprehensive framework for the regularization of adjoint analysis in multiscale PDE systems", Journal of Computational Physics 195 (1), 49-89, 2004.

[19] J. Jocobsen, "As Flat As Possible", SIAM Review 49, 491-507, (2007).

[20] A. Chambolle, R. A. DeVore, N.Lee, and B. J. Lucier, "Nonlinear Wavelet Image Processing: Variational Problems, Compression, and Noise Removal through Wavelet Shrinkage", IEEE Transactions on Image Processing, 7, 319-355, (1998).

[21] M. Farge, "Wavelet Transforms and their Applications to Turbulence", Annual Review of Fluid Mechanics 24, 395-457, (1992).

[22] T. R. Bewley and B. Protas, "Skin friction and pressure: the "footprints" of turbulence", Physica D 196, 28-44, (2004).

[23] J. M. Hyman and B. Nicolaenko, "The Kuramoto-Sivashinsky equation: a bridge between PDEs and dynamical systems", Physica D 18, 113-126, (1986).

[24] R. Temam, "Infinite-Dimensional Dynamical Systems in Mechanics and Physics", Applied Mathematical Sciences 68 (2nd edition), Springer, (1997).

[25] R. A. Adams and J. F. Fournier, "Sobolev Spaces”, Elsevier, (2005). 
[26] Ch. Hirsch, "Numerical Computation of Internal and External Flows", John Wiley \& Sons, 1989.

[27] R. M. Lewis, "A nonlinear programming perspective on sensitivity calculations for systems governed by state equations”, ICASE Technical Report TR-97-12, (1997).

[28] I. W. Knowles, "Descent methods for inverse problems", Nonlinear Analysis 47 32353245, (2001).

[29] H. Triebel, “Theory of Function Spaces II”, Birkhäuser, (1992).

[30] G. P. Galdi, "An Introduction to the Mathematical Theory of the Navier-Stokes Equations. Volume I: Linearized Steady Problems", Springer, (1994).

[31] C. T. Kelley, "Solving Nonlinear Equations with Newton's Method”, SIAM, (2003).

[32] S. Mallat, "Une exploration des signaux en ondelettes", Les Éditions de l'École Polytechnique, (2000).

[33] A. Cohen, "Numerical Analysis of Wavelet Methods", North-Holland, (2003).

[34] J. Buckheit, S. Chen, D. Donoho, I. Johnstone, Jeffrey Scargle, "About Wavelab", available at http://www-stat.stanford.edu/ wavelab, (2005).

[35] H. Choi and R. G. Baraniuk, "Multiple wavelet basis image denoising using Besov ball projections" IEEE Signal Processing Letters 11, 717-720, (2004).

[36] W. H. Press, B. P. Flanner, S. A. Teukolsky and W. T. Vetterling, "Numerical Recipes: the Art of Scientific Computations", Cambridge University Press, Cambridge, (1986).

[37] M. Chevalier, J. Hœpffner, T. Bewley and D. S. Henningson, "State estimation in wallbounded flow systems. Part 2. Turbulent flows", Journal of Fluid Mechanics 552 167187, (2006). 